\title{
Title: Social hosts evade predation but have deadlier parasites
}

\author{
Authors: Jason C. Walsman ${ }^{\mathrm{a}, *}$, Mary J. Janecka ${ }^{\mathrm{a}}$, David R. Clark ${ }^{\mathrm{a}}$, Rachael D. Kramp ${ }^{\mathrm{a}}$, Faith \\ Rovenolt $^{\mathrm{a}}$, Regina Patrick ${ }^{\mathrm{a}}$, Ryan S. Mohammed ${ }^{\mathrm{b}}$, Mateusz Konczal ${ }^{\mathrm{c}}$, Clayton E. Cressler ${ }^{\mathrm{d}}$, \\ Jessica F. Stephenson ${ }^{\mathrm{a}, *}$
}

\section{Affiliations:}

${ }^{a}$ Department of Biological Sciences, University of Pittsburgh; Pittsburgh, PA USA.

*Corresponding author. Email: walsmanjason@gmail.com; jess.stephenson@pitt.edu

${ }^{b}$ Department of Life Sciences, The University of the West Indies; St. Augustine, Trinidad and Tobago.

${ }^{c}$ Evolutionary Biology Group, Faculty of Biology, Adam Mickiewicz University; 60-614 Poznań, Poland.

${ }^{\mathrm{d}}$ School of Biological Sciences, University of Nebraska; Lincoln, NE USA.

\begin{abstract}
Parasites exploit hosts to replicate and transmit, but overexploitation kills host and parasite (1): predators may shift this cost-benefit balance by consuming hosts (2-4) or changing host behavior, but the strength of these effects remains unclear. Modeling both, we find a primary, strong effect: hosts group to defend against predators (5), increasing parasite transmission, thus multiple infections, and therefore favoring more exploitative, virulent, parasites (6). Indeed, among 18 Trinidadian Gyrodactyus spp. parasite lines, those collected from high predation guppy populations were more virulent in common garden than those from low
\end{abstract}


predation populations. Our model accurately predicted this result when parametrized with our experimentally demonstrated virulence-transmission trade-off, implicating the behavioral effects of predation. Broadly, our results indicate that reduced social contact selects against parasite virulence.

One-Sentence Summary: Our theory and data show predators cause increased host social grouping; the resulting transmission favors parasite virulence. 
Main text: Unprecedented infectious disease emergence among human and wildlife populations (7) demands that we improve our understanding of the evolutionary ecology of parasites and pathogens (hereafter 'parasites'). A key tenet of the evolutionary theory of infectious disease is that parasites face a trade-off between virulence and transmission $(8,9)$. Parasites exploit their hosts to replicate and transmit but increasing this exploitation harms parasite fitness by killing hosts ('virulence'), which reduces the time window for successful transmission, or the 'infectious period' (1). With appropriate curvature, this trade-off should result in stabilizing selection for intermediate virulence (10-13). While theory predicts various mechanisms can shift the costs and benefits of exploitation, altering virulence evolution $(2-4,6)$ and host disease outcomes $(9,12$, 14, 15), empirical evaluation of their relative importance remains scant $(9,16,17)$.

Predation, a ubiquitous ecological interaction, can theoretically select for higher or lower virulence $(2,4,9)$ through multiple pathways (we highlight four in Fig. 1). Predation directly increases host mortality, selecting for faster exploitation and transmission before the host dies, and hence increased virulence (pathway 1 in Fig. 1; 2, 3, 17). Conversely, predation selects for lower virulence by reducing the density of infected hosts, reducing the per-host rate at which new infections arise ('force of infection'); lower force of infection reduces infections with multiple parasite genotypes ('multiple infections') and thus the within-host competition between parasite genotypes that favors higher virulence (pathway 2 in Fig. 1; 4, 16). In addition to these consumptive effects, predators can also affect host traits (18), non-consumptively shifting how hosts interact with their parasites (19-21), and probably virulence evolution. One example is host grouping, a common defense (5) which effectively decreases predator-induced mortality (22), potentially selecting for lower virulence (pathway 3 in Fig. 1). However, grouping rate also increases host-host contact rate, increasing the force of infection, so host grouping could increase multiple infections and therefore select for higher virulence (pathway 4; 6, 16, 23). Both 
consumptive and non-consumptive effects of predation act simultaneously in natural communities (20), but their relative importance for selection on parasite virulence lacks empirical and theoretical clarification.

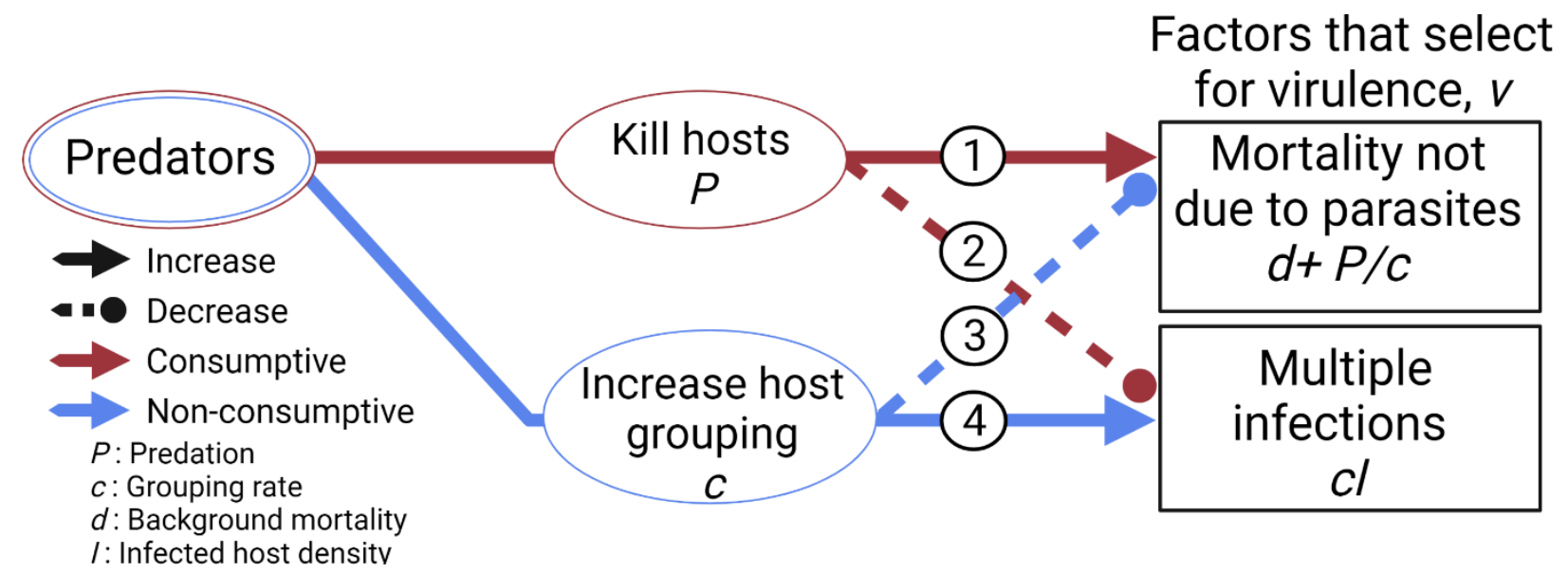

Figure 1. Predators alter selection on virulence through consumptive and non-consumptive

pathways. The consumptive (red) and non-consumptive (blue) effects of predation

simultaneously act to increase (solid, triangular arrows) and decrease (dashed, rounded arrows) factors that select for higher virulence. Mortality not due to parasites is $d+P / c$ while the force of infection for multiple infections is proportional to $c I$. Host grouping rate, $c$, controls host-host contact rate for transmission.

\section{Scope of study}

Here, we elucidate how predators affect virulence using theory and data from Trinidadian guppies, Poecilia reticulata and their Gyrodactylus spp. parasites (Fig. 2). Persistent, natural variation in predation risk drives population-level variation in guppy grouping rate ('shoaling rate') (24-26), probably influencing transmission of Gyrodactylus spp. ectoparasites (27-30): high-predation populations shoal more and suffer higher Gyrodactylus spp. infection prevalence 
$(28,31,32)$. First, we test for the commonly-assumed virulence-transmission trade-off and multiple infections (Table S2). We then use laboratory and field measurements to parametrize a model with selection on host and parasite phenotypes interacting with host and parasite densities ('eco-coevolutionary model'). Finally, we test model predictions against wild-collected, Gyrodactylus spp. parasites, assaying parasite virulence in common garden conditions. The common garden isolated differences in parasite exploitation of hosts while keeping host defenses constant. 


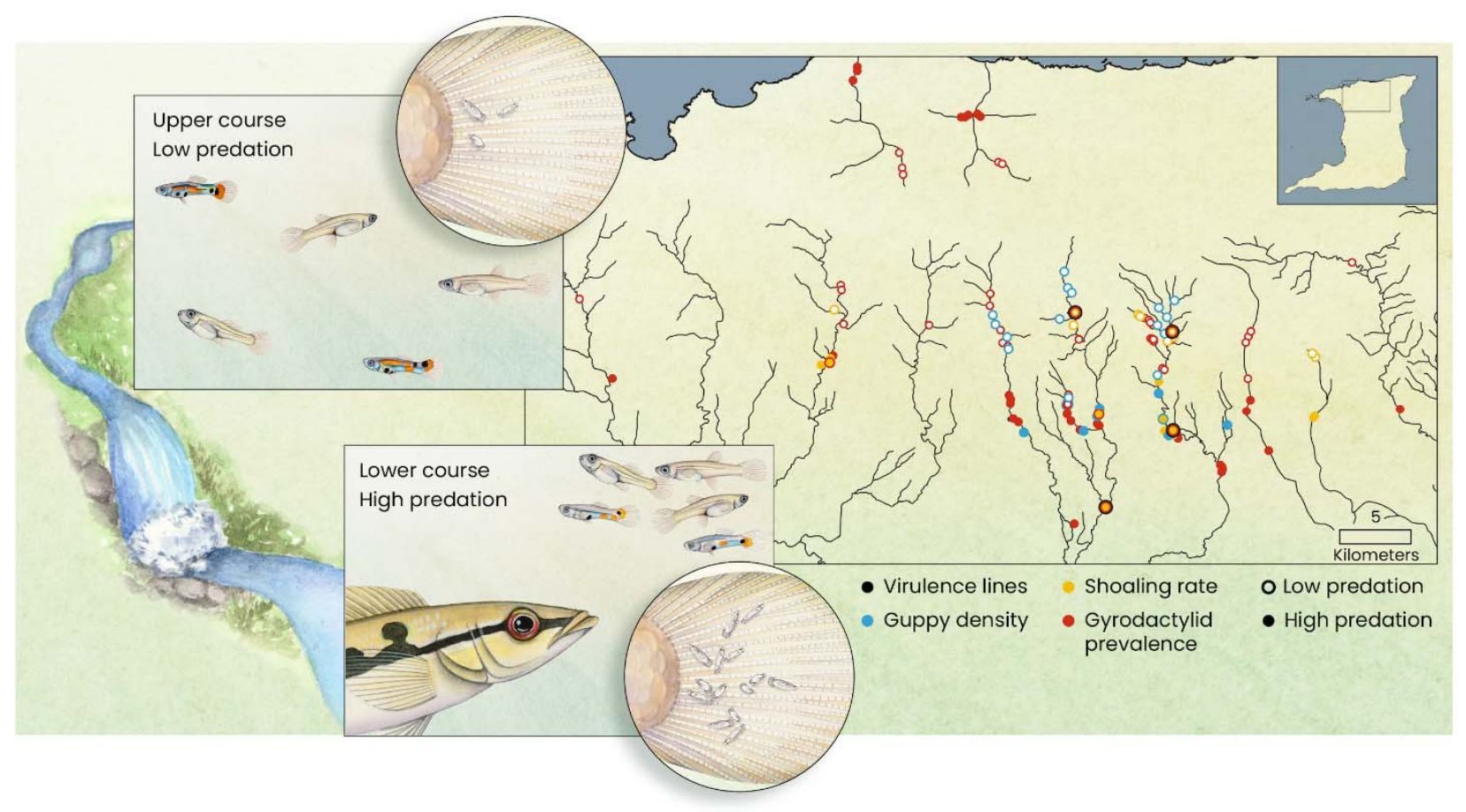

Figure 2. Natural guppy populations differ in predation, driving evolutionary divergence in

shoaling rate. Waterfalls divide upper and lower course guppy populations, preventing upstream migration of large piscivores. Natural populations have therefore evolved under different predation regimes, replicated across rivers. Shoaling rate differences apparently drive population-level differences in transmission rate of (28-30), and thus selection on the virulence of, their highly prevalent, directly transmitted monogenean ectoparasites Gyrodactylus spp.. The map shows locations and data types that parameterized and validated model quantities [see Materials and Methods]. To focus on the various effects of predation, we used data from a river if that data type was available in low- and high-predation populations for that river. 


\section{Results}

Gyrodactylus spp. transmission trades off with virulence, mediated by parasite intensity
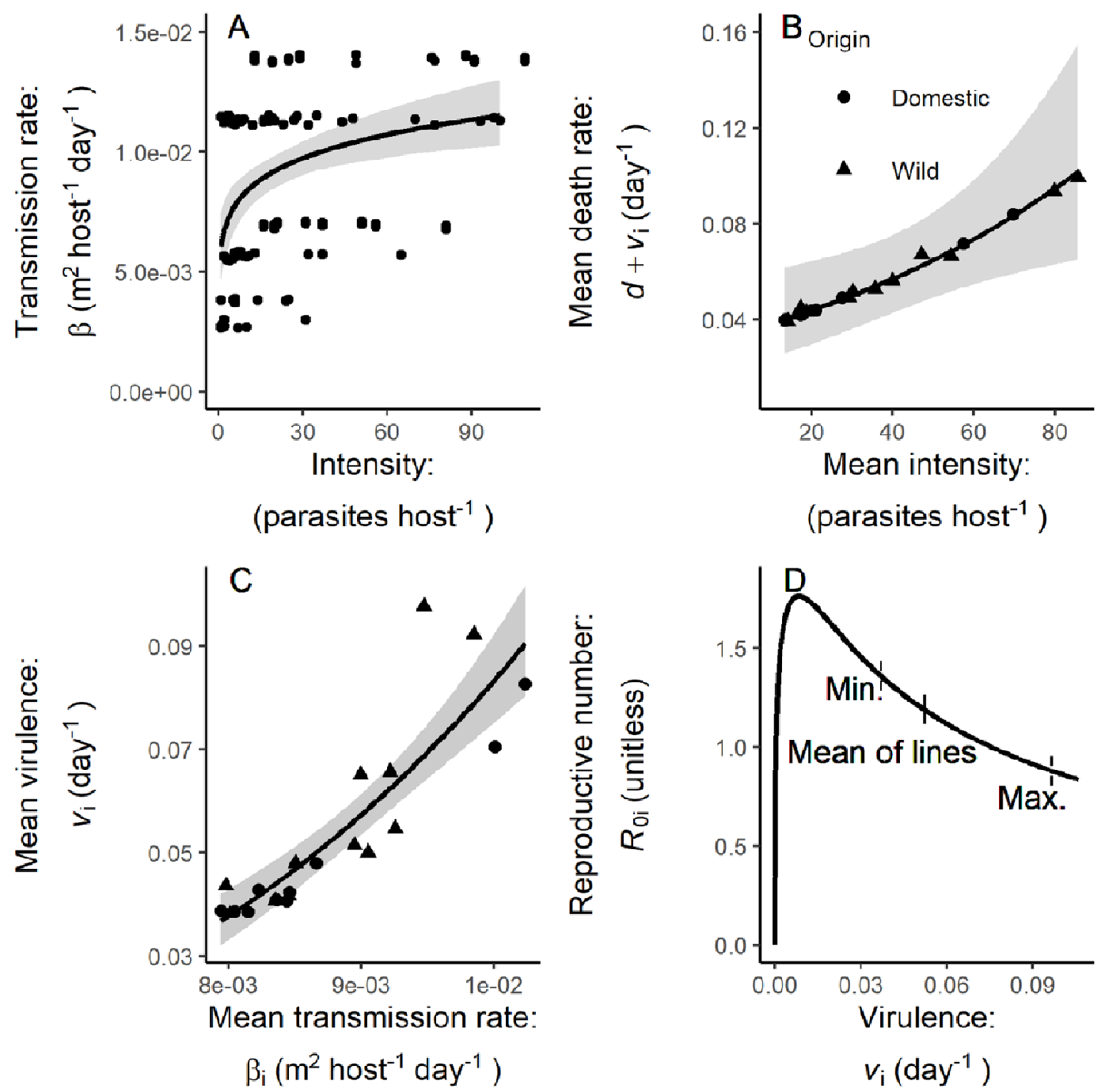

Figure 3. Infection intensity links transmission rate and virulence with a stabilizing trade-

off. (A) Across four parasite lines, transmission rate increased with infection intensity. Points are transmission events jittered vertically. (B) Across 22 ['domestic' (circles) or 'wild' (triangles)] parasite lines, higher intensity (a measure of parasite growth rate) induced higher host death rate $\left(d+v_{i}\right.$, a measure of virulence; back-transformed partial residuals). (C) (A) allows a link between 
transmission rate and virulence across lines. Points in $(\mathrm{B}, \mathrm{C})$ are mean line traits. In $(\mathrm{A}-\mathrm{C})$, bands are $95 \%$ C.I. (D), The curvature in (C) maximizes $\mathrm{R}_{0 \mathrm{i}}$ at intermediate transmission rate/virulence.

We found a virulence-transmission trade-off among Gyrodactylus spp. lines by measuring disease traits on individual hosts. First, we found that transmission rate from a donor to sentinel host increased asymptotically as intensity increased [Fig. $3 \mathrm{~A} ; \beta=6.08 \times 10^{-3}$ intensity $^{0.138}$; Generalized linear model (GLM) $N=101, P=3.48 \times 10^{-5}, r=0.35$; see Materials and Methods for more details]. This pattern did not differ across two experiments using different, domestic parasite lines (isolated from commercial guppies instead of wild-caught; line effect: GLM, $N=101, P=0.596, \varphi=0.12)$.

Second, we measured worm parasites per fish (intensity) and the death rate of infected hosts for 22 laboratory-maintained lines (including three of four from Fig. 3A). These lines differed in the mean intensity they attained (ANOVA, $N=1171, P=9.36 \times 10^{-25}, \eta^{2}=0.13$ ), likely due to faster exploitation of and reproduction on individual hosts; higher intensity lines imposed a higher mean death rate on infected hosts (Fig. 3B; GLM, $N=22, P=0.008, r=0.49$ ). Across intensities, domestic lines imposed less death (GLM, $N=22, P=1.24 \times 10^{-4}, r=0.63$; domestic $=0.016 v s$. wild $=0.052$ at overall mean intensity). We used back-transformed, partial residual death rate to control for non-focal predictors (line origin: Fig. 3B shows the relationship as if all lines were wild) so that we could determine the relationship relevant to wild parasites (all results are very similar if instead we set all lines as domestic, see Supplementary Code).

Third, we predicted the mean transmission rate for each line, given its intensity measurements and the transmission-intensity relationship in Fig. 3A. We found that the mean transmission rate for each line traded-off with the mean virulence (we get virulence, $v$, from infected host death rate, $d+v$, given $d=1.30 \times 10^{-3}$ day $^{-1}$ ): parasite lines that attained higher 
intensity benefitted from higher transmission rate but higher virulence shortened their infections, on average (Fig. 3C). Since Gyrodactylus spp. are capable of sexual and asexual reproduction, we tested whether any of our lines were clones (i.e., identical multi-locus genotypes, MLGs). Genotyping a subset (13/22, based on sample availability) found no identical MLGs (all pairs within populations differed at $\geq 16$ loci, representing at least half of all variable loci for each source population, see Table S2).

The curvature of this trade-off indicated stabilizing selection on virulence (GLM fit: $v_{\mathrm{i}}=$ $1.38 \times 10^{6} \beta_{\mathrm{i}}^{3.61} ; v_{\mathrm{i}}$ and $\beta_{\mathrm{i}}$ are average traits for a line). Bootstrapping parasite lines included in the analysis showed this curvature was significant (exponent $>1$ in all $10^{4}$ bootstrapped samples) and AIC indicates a better fit to the data than for a linear model $(\triangle \mathrm{AIC}=13.3)$. Theory predicts that the trade-off curvature leads to optimal parasite fitness at intermediate transmissibility $\left(\beta_{\mathrm{i}}=\right.$ $c T_{\mathrm{i}}, c$ is host shoaling rate, $T_{\mathrm{i}}$ is a line's transmissibility) and virulence [Fig. 3D; $R_{0 \mathrm{i}}=$ $c T_{\mathrm{i}} S /\left(d+v_{\mathrm{i}}+y\right)$ for pure infections where we use reasonable values of $S=10$ susceptible host $\mathrm{m}^{-2}$ and $\gamma=0.020 \mathrm{day}^{-1}$, see Table 1]. Unsurprisingly, parasite lines fell to the right of this optimum: higher background mortality in wild than laboratory guppies shifts the optimum higher; the mixed-stock guppies in our common garden assay may differ substantially from local, wild hosts; and multiple infections (common in the wild, Table S1) select for higher virulence than would optimize $R_{0 \mathrm{i}}$ of pure infections (4).

\section{Eco-coevolutionary model resolves complexity to show that social hosts have deadlier parasites}

Our model of predators that shift coevolution of host shoaling rate and parasite virulence provides two general insights. First, it demonstrates that the net, selective effect of predation, shoaling rate, or other ecological factors on virulence evolution depends only on their effect on the rate at which infections are lost to mortality or multiple infections (inverse of infectious 
period; see Supplementary Text for proof). Second, the model shows that shoaling rate evolves to balance predator-induced mortality against parasite-induced mortality. When predation increases, hosts evolve higher shoaling rate, increasing transmission rate. When parasites are more abundant and virulent, hosts evolve lower shoaling rate to prevent infection. For some parameter values, higher virulence can select for higher shoaling rate if parasites become so virulent that the density of infected hosts and force of infection decline strongly (Fig. S1).

When parametrized with empirical data from the guppy-Gyrodactylus spp. system, the model complexity resolves into one dominant pathway: predation increases co-evolutionarily stable contact rate, multiple infections, and thus virulence (Fig. 4; strength of pathways from Fig. 1 : pathway $1=0.131$, pathway $2=-0.166$, pathway $3=-0.063$, pathway $4=1.18$; see Supplementary Text for derivation). When predators only have consumptive effects on predation (i.e., hosts do not evolve; pathways 1 and 2), predation decreases prevalence and virulence (grey curves in Fig. 5A-E; presence/absence of parasite evolution has little effect on grey curves). However, as hosts evolve higher shoaling rate in response to predation (Fig. 5A), predation also increases prevalence (black curve in Fig. 5B) in all tested parameter sets (see sensitivity analysis methods). Increasing predation increases overall host mortality and reduces host density, particularly when hosts evolve (Fig. 5C). Further, most of the increase in mortality (76\%) with predation is due to increased parasite-induced mortality (prevalence $\times$ virulence), rather than increased predator-induced mortality (Fig. S2; robust to parameters: Fig. S3). Higher shoaling rate increases the force of infection (and thus multiple infections), selecting for higher parasite intensity and virulence (Figs. 5D, E; note intensity was not modelled directly but back-calculated from transmissibility according to Fig. 3B); in all parameter sets, higher predation increased virulence. Importantly, predators increase virulence through their non-consumptive effects on shoaling rate (across parameter sets, see Fig. S3). 


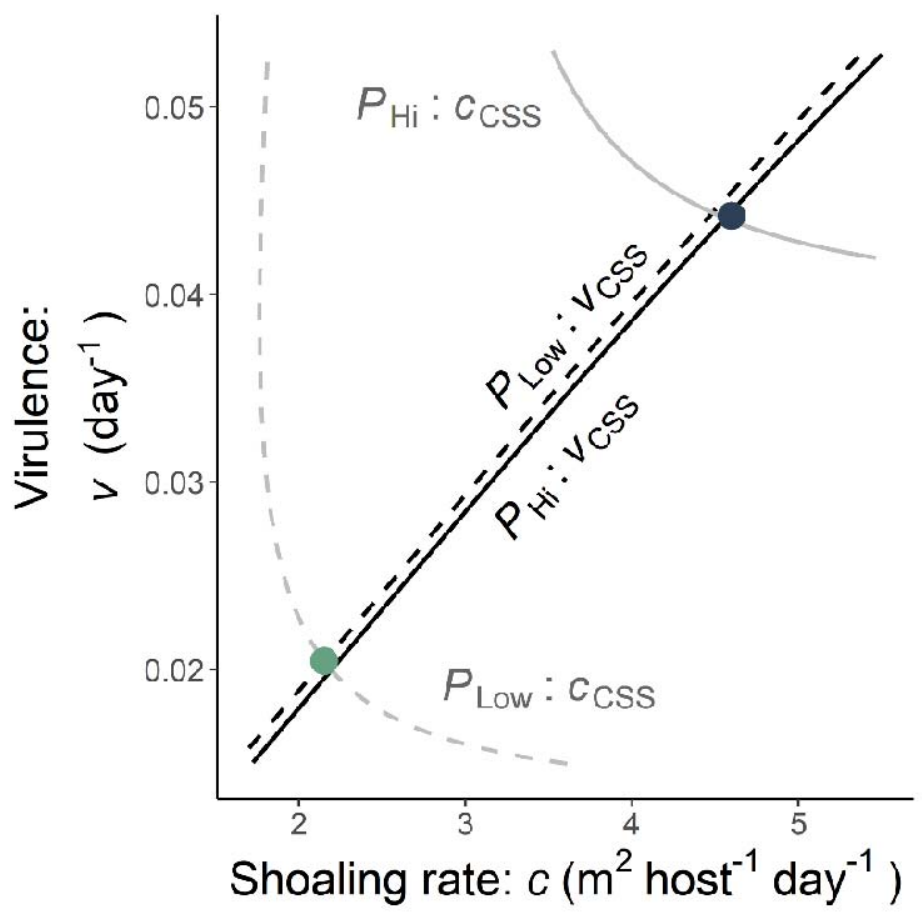

Figure 4. Predation drives increased shoaling rate and virulence in the eco-coevolutionary

model. Curves give the continuously stable strategy (CSS) for parasite $\left(v_{\mathrm{CSS}}\right.$ black) or host $\left(c_{\mathrm{CSS}}\right.$ grey) evolution at predation levels fitted to correspond to natural populations $\left(P_{\text {Low }}\right.$; dashed or $P_{\mathrm{Hi}}$; solid). Parasites evolve higher virulence with higher shoaling rate. The consumptive effects of predation have little, net effect on evolved virulence. Hosts evolve lower shoaling rate with increasing virulence (grey curves move toward lower $c$ as $v$ increases). Predation substantially increases $c_{\text {CSS }}$. Host and parasite curves intersect at coevolutionarily stable points (green and blue points). As predation increases (from green point to blue point), coevolutionarily stable shoaling rate $\left(c_{\text {coCss }}\right)$ and virulence $\left(v_{\text {coCSS }}\right)$ increase. 
bioRxiv preprint doi: https://doi. org/10.1101/2021.09.10.459661. this version posted October 192021 . The copyright holder for this preprint (which was not certified by peer review) is the author/funder, who has granted bioRxiv a license to display the preprint in perpetuity. It is made available under aCC-BY-NC-ND 4.0 International license.

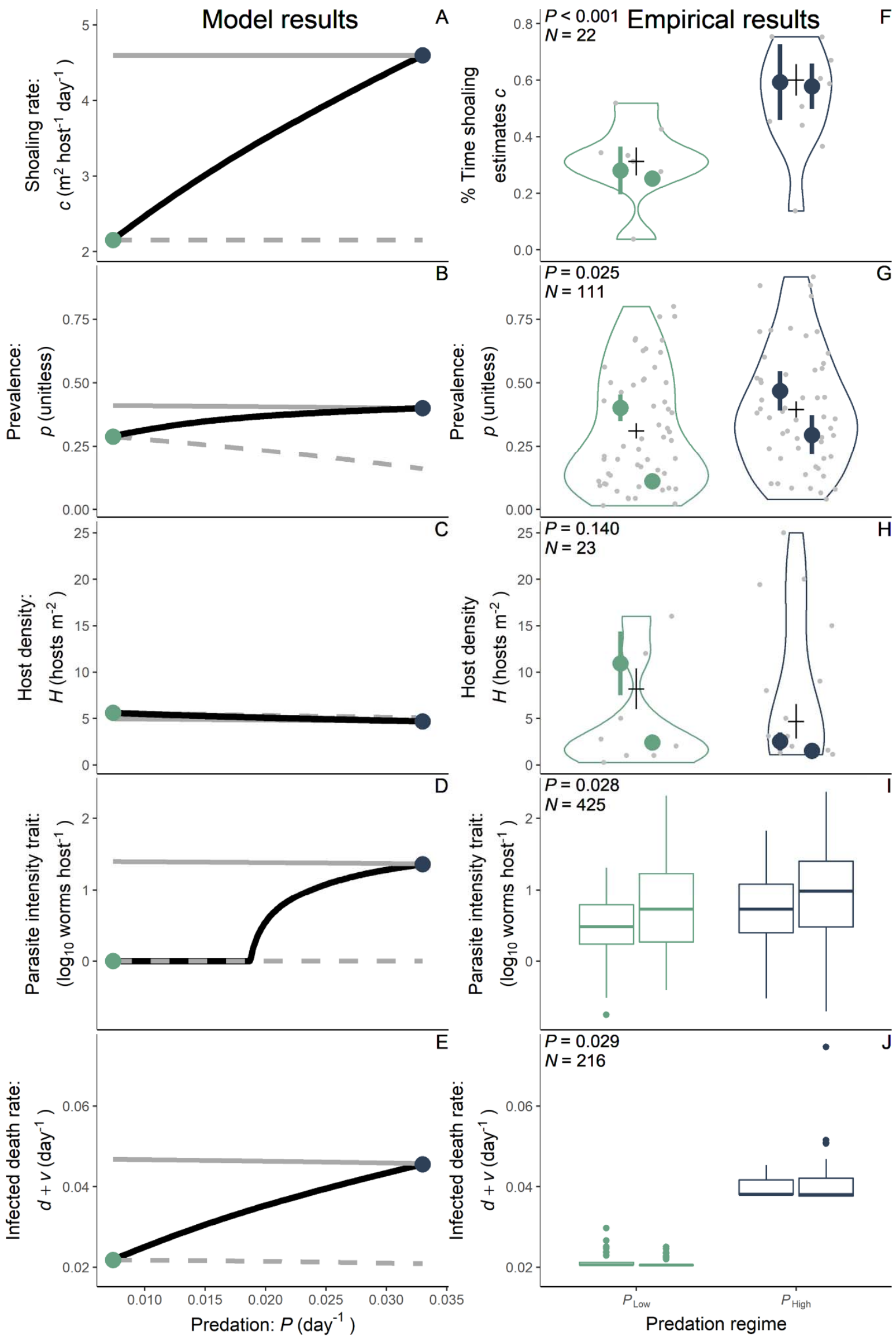


Figure 5. Predation increases shoaling rate and thus selects for higher virulence. (A-E) Model results. Black curves: eco-coevolution (connecting Fig. 4 colored points). Grey curves: no host evolution. (F-H) Empirical training data. Horizontal segments: predation regime means. Grey points: one river/regime/year mean. Colored points: one focal river/predation regime mean (Aripo left of Guanapo). Vertical bars: standard errors. Training data: violins. Validating data (I, J): boxplots (back-transformed partial residuals; center line, median; box limits, first and third quartiles; whiskers, $1.5 \mathrm{x}$ interquartile range; points, outliers). $P$-values for the effect of course and sample sizes $(N)$ provided. (A, F) Shoaling increased with predation. (B, G) Prevalence increased with predation while $(\mathrm{C}, \mathrm{H})$ host density decreased non-significantly. (D, I) Parasite intensity [note, we do not model intensity $\operatorname{lower}$ than $\log (1$ worm $)=0$ ] and $(\mathrm{E}, \mathrm{J})$ virulence increased with predation.

\section{Empirical results quantitatively match model predictions that social hosts have deadlier}

\section{parasites}

The model's coevolutionary outcomes successfully fit training data from our collections and the literature (Fig. 5A-C, F-H; table 1). High predation fish spent more time shoaling in our assay (Generalized Linear Mixed Model [GLMM], $N=68, P=0.006, r=0.32$ ) and across population estimates extracted from the literature (GLMM, $N=22, P=8.31 \times 10^{-4}, r=0.58$; Fig. 5F). This helps explain why high predation populations suffered higher parasite prevalence across multiple rivers, sites, and years (GLMM, $N=107, P=0.025, r=0.21$; Fig. 5G). Higher levels of predation and parasitism may depress host density (though not significantly so: GLMM, $N=23, P=0.140, r=-0.29$; Fig. 5H). Overall, the model fit the training data reasonably well, with an average relative error of $10.5 \%$ per fitted quantity (shown in Fig. 5A-C, F-H and Table 1). 
Alternative models do not fit the data as well, bolstering confidence in our focal ecocoevolutionary model. A model without host evolution (only consumptive effects of predators) fits the data poorly (relative error of $15.2 \%$ per fitted quantity, see Fig. S4) despite having an additional free parameter. A different alternative model fits an exponent governing how effectively shoaling protects from predation (exponent $=1$ in our focal model) but the miniscule shift in model fit (relative error improves from $10.4 \%$ to $10.1 \%$, see Fig. S4) does not justify the additional free parameter. Accounting for model complexity, our focal model provided the best fit to the training data, and of the three models it thus likely best captures the consumptive and non-consumptive effects of predation.

We tested the focal model's trained predictions by quantifying the traits of 18 parasite lines isolated from wild populations and maintained in the lab under common garden conditions for 65 days. Lines from high predation populations attained higher intensity on infected, mixedstock hosts (GLMM, $N=425, P=0.028, r=0.11$; Fig. 5I) and induced higher death rate (GLMM, $N=216, P=0.029, r=0.15$; Fig. 5J). The two parasite species, $G$. turnbulli (11 known lines) and G. bullatarudis (3 known lines) did not differ significantly in intensity (GLMM, $N=$ $345, P=0.098, r=0.09$ ) or virulence [GLM, $N=177, P=0.345, r=0.07$; unlike (33)]. Restricting the analysis for Fig. 5J to G. turnbulli found marginally higher intensity (GLM, $N=$ 208, $P=0.076, r=0.12$ ) and significantly higher virulence in high predation populations (GLM, $N=102, P=0.009, r=0.25)$. The overall quantitative match between the model-predicted mortality and our empirical results supports our model's inferences $(d+v$ in the model $v s$. mean of $d+v$ back-transformed partial residuals: low predation $0.020 v s .0 .021$; high predation: 0.044 vs. 0.041). We compared theoretical predictions to partial residuals to control for non-focal predictors in the statistical model, especially duration of parasite maintenance in the laboratory, to obtain the most biologically relevant estimate of virulence. Our theoretical model makes this 
prediction based on higher shoaling rate increasing multiple infections, which seem more frequent in high predation populations on a similar scale to shoaling rate (shoaling rate $\sim 2$ times higher and coinfection $\sim 3$ times higher in high predation; Table S1).

The model-data agreement on evolved virulence aids model-data agreement on how mortality changes across predation regime. In our model, $76 \%$ of the increased mortality across predation regime is increased parasite-induced mortality while increased predator-induced mortality accounts for $24 \%$. Prevalence in natural populations and virulence data on laboratory fish $\left(p_{\mathrm{Hi}} v_{\mathrm{Hi}}-p_{\mathrm{Low}} v_{\mathrm{Low}}\right)$ indicated that parasitism would explain $64 \%$ of the mortality difference from Reznick et al (34) while predation may account for the remaining $36 \%$.

Discussion. Our theoretical-empirical approach clarifies that predation drives the evolution of parasite virulence by increasing host shoaling rate. As a result, shoaling in response to increasing predation pressure leads to parasite-induced mortality rising more than that induced by predators. In contrast to these strong non-consumptive effects, we found that the consumptive effects of predation are small, balance one another out, and barely alter virulence evolution. To our knowledge, our study is the first to model the non-consumptive effects of predation on virulence evolution. Further, we used data to train and test a model that infers and compares the strength of consumptive and non-consumptive effects in natural populations. We discuss key emergent patterns.

Theory shows that shoaling rate drives virulence evolution depends on multiple infections. Without multiple infections, shoaling decreases predator-induced mortality and thus selects for decreased virulence (35). With multiple infections, we and others (6) find that increased grouping rate selects for higher virulence. Empirically, we do not know of previous tests of the effect of host-host contact rate on virulence evolution, but our result is analogous to 
more host dispersal $(15,36,37)$ and parasite founder diversity (14) selecting for higher host exploitation through high local parasite diversity (38).

We assayed parasite traits using infections on mixed-stock, wildtype guppies: this approach allowed us to draw robust conclusions about parasite evolution but leaves untested how host defenses, other than shoaling rate, may affect disease dynamics in natural communities. Thus far, guppy defenses against parasites have not been robustly characterized across predation regime. Illustrative data suggest that, in our focal Guanapo river (experimental test; 39), and perhaps more generally (field surveys; 28, 31), guppies from low predation regimes are better defended against Gyrodactylus spp.. Low predation guppies may be better able to evolve in response to Gyrodactylus spp.: they need not balance this investment with defense against predators (22), and sexual selection, which acts on gyrodactylid resistance (40), is more effective in the absence of predators (41). However, in our focal Aripo river, low predation guppies appear less resistant than high predation guppies (experimental test; 42). Despite this apparent difference between our focal rivers, our measured virulence matched our model predictions for both, suggesting the patterns we observe may be robust to population-level differences in host defenses. Nevertheless, how host behavioral and immunological defenses may coevolve with parasite virulence in natural communities remains an outstanding and complex theoretical as well as empirical challenge.

The importance and implications of predation and social parasite transmission, evident from our model and data, may hold across directly transmitted parasites of group-living hosts (43). Predators drive defensive group-living in animals across taxa (5), which can increase parasitism, creating ecological and evolutionary feedbacks between host sociality and parasites (43). Parasites evolve along virulence-transmission trade-offs in systems ranging from viruses of humans $(10,13)$, bacterial pathogens of birds (11), protozoan pathogens of insects (12), and our 
monogenean fish ectoparasite. Multiple infections are common for many parasites, allowing the simple mechanism of competition for within-host resources to select for higher virulence (16).

Thus, diverse systems likely meet the essential assumptions of our model: predators may frequently shift the antagonistic interplay of host sociality and parasite virulence, driving hosts into the arms of more virulent parasites. Conversely, these results also indicate that social distancing may select for lower virulence when parasites exhibit multiple infections and a virulence-transmission trade-off (e.g., influenza A virus; 10, 44). Host behavior that reduces contact may thus effectively control both the spread and virulence evolution of pathogens and parasites.

\section{References and Notes}

1. R. M. Anderson, R. M. May, Coevolution of hosts and parasites. Parasitology 85, 411426 (1982).

2. K. Choo, P. D. Williams, T. Day, Host mortality, predation and the evolution of parasite virulence. Ecology letters 6, 310-315 (2003).

3. P. D. Williams, T. Day, Interactions between sources of mortality and the evolution of parasite virulence. Proceedings of the Royal Society of London. Series B: Biological Sciences 268, 2331-2337 (2001).

4. S. Gandon, V. A. Jansen, M. Van Baalen, Host life history and the evolution of parasite virulence. Evolution 55, 1056-1062 (2001).

5. J. Krause, G. D. Ruxton, G. Ruxton, I. G. Ruxton, Living in groups. (Oxford University Press, 2002).

6. F. Prado, A. Sheih, J. D. West, B. Kerr, Coevolutionary cycling of host sociality and pathogen virulence in contact networks. Journal of theoretical biology 261, 561-569 (2009).

7. M. Everard, P. Johnston, D. Santillo, C. Staddon, The role of ecosystems in mitigation and management of Covid-19 and other zoonoses. Environmental science \& policy 111, 7-17 (2020).

8. S. Alizon, A. Hurford, N. Mideo, M. Van Baalen, Virulence evolution and the trade $\square$ off hypothesis: history, current state of affairs and the future. Journal of evolutionary biology 22, 245-259 (2009).

9. C. E. Cressler, D. V. McLeod, C. Rozins, J. Van Den Hoogen, T. Day, The adaptive evolution of virulence: a review of theoretical predictions and empirical tests. Parasitology 143, 915-930 (2016).

10. B. McKay, M. Ebell, A. P. Dale, Y. Shen, A. Handel, Virulence-mediated infectiousness and activity trade-offs and their impact on transmission potential of influenza patients. Proceedings of the Royal Society B 287, 20200496 (2020). 
11. C. Bonneaud et al., Experimental evidence for stabilizing selection on virulence in a bacterial pathogen. Evolution Letters, (2020).

12. J. C. De Roode, A. J. Yates, S. Altizer, Virulence-transmission trade-offs and population divergence in virulence in a naturally occurring butterfly parasite. Proceedings of the National Academy of Sciences 105, 7489-7494 (2008).

13. C. Fraser, T. D. Hollingsworth, R. Chapman, F. de Wolf, W. P. Hanage, Variation in HIV-1 set-point viral load: epidemiological analysis and an evolutionary hypothesis. Proceedings of the National Academy of Sciences 104, 17441-17446 (2007).

14. E. A. Herre, Population structure and the evolution of virulence in nematode parasites of fig wasps. Science 259, 1442-1445 (1993).

15. M. Boots, M. Mealor, Local interactions select for lower pathogen infectivity. Science 315, 1284-1286 (2007).

16. S. Alizon, J. C. de Roode, Y. Michalakis, Multiple infections and the evolution of virulence. Ecology letters 16, 556-567 (2013).

17. J. J. Bull, A. S. Lauring, Theory and empiricism in virulence evolution. PLoS pathogens 10, e1004387 (2014).

18. S. D. Peacor, E. E. Werner, The contribution of trait-mediated indirect effects to the net effects of a predator. Proceedings of the National Academy of Sciences 98, 3904-3908 (2001).

19. O. Seppälä, A. Karvonen, E. T. Valtonen, Shoaling behaviour of fish under parasitism and predation risk. Animal Behaviour 75, 145-150 (2008).

20. L. K. Lopez, M. A. Duffy, Mechanisms by which predators mediate host-parasite interactions in aquatic systems. Trends in Parasitology, (2021).

21. M. C. Rigby, J. Jokela, Predator avoidance and immune defence: costs and trade-offs in snails. Proceedings of the Royal Society of London B: Biological Sciences 267, 171-176 (2000).

22. J.-G. J. Godin, Antipredator function of shoaling in teleost fishes: a selective review. Naturaliste Canadien 113, 241-250 (1986).

23. S. Gandon, M. van Baalen, V. A. Jansen, The evolution of parasite virulence, superinfection, and host resistance. The American Naturalist 159, 658-669 (2002).

24. A. E. Magurran, Evolutionary ecology: the Trinidadian guppy. (Oxford University Press on Demand, 2005).

25. A. E. Magurran, B. H. Seghers, Variation in schooling and aggression amongst guppy (Poecilia reticulata) populations in Trinidad. Behaviour, 214-234 (1991).

26. B. H. Seghers, A. E. Magurran, Predator inspection behaviour covaries with schooling tendency amongst wild guppy, Poecilia reticulata, populations in Trinidad. Behaviour 128, 121-134 (1994).

27. M. Huizinga, C. Ghalambor, D. Reznick, The genetic and environmental basis of adaptive differences in shoaling behaviour among populations of Trinidadian guppies, Poecilia reticulata. Journal of evolutionary biology 22, 1860-1866 (2009).

28. J. F. Stephenson, C. Van Oosterhout, R. S. Mohammed, J. Cable, Parasites of Trinidadian guppies: evidence for sex $\square$ and age $\square$ specific trait $\square$ mediated indirect effects of predators. Ecology 96, 489-498 (2015).

29. E. L. Richards, C. Van Oosterhout, J. Cable, Sex-specific differences in shoaling affect parasite transmission in guppies. PLoS One 5, e13285 (2010).

30. M. B. Johnson, K. D. Lafferty, C. Van Oosterhout, J. Cable, Parasite transmission in social interacting hosts: monogenean epidemics in guppies. PLoS One 6, (2011). 
31. K. M. Gotanda et al., Adding parasites to the guppy-predation story: insights from field surveys. Oecologia 172, 155-166 (2013).

32. B. A. Fraser, I. W. Ramnarine, B. D. Neff, Temporal variation at the MHC class IIB in wild populations of the guppy (Poecilia reticulata). Evolution: International Journal of Organic Evolution 64, 2086-2096 (2010).

33. J. Cable, C. Van Oosterhout, The impact of parasites on the life history evolution of guppies (Poecilia reticulata): the effects of host size on parasite virulence. International journal for parasitology 37, 1449-1458 (2007).

34. D. N. Reznick, M. J. Butler IV, F. H. Rodd, P. Ross, Life $\square$ history evolution in guppies (Poecilia reticulata) 6. Differential mortality as a mechanism for natural selection. Evolution 50, 1651-1660 (1996).

35. M. H. Bonds, D. C. Keenan, A. J. Leidner, P. Rohani, Higher disease prevalence can induce greater sociality: a game theoretic coevolutionary model. Evolution 59, 1859-1866 (2005).

36. B. Kerr, C. Neuhauser, B. J. Bohannan, A. M. Dean, Local migration promotes competitive restraint in a host-pathogen'tragedy of the commons'. Nature 442, 75-78 (2006).

37. M. Boots, A. Sasaki, 'Small worlds' and the evolution of virulence: infection occurs locally and at a distance. Proceedings of the Royal Society of London. Series B: Biological Sciences 266, 1933-1938 (1999).

38. G. Wild, A. Gardner, S. A. West, Adaptation and the evolution of parasite virulence in a connected world. Nature 459, 983-986 (2009).

39. F. Dargent, G. Rolshausen, A. Hendry, M. Scott, G. Fussmann, Parting ways: parasite release in nature leads to sex $\square$ specific evolution of defence. Journal of evolutionary biology 29, 23-34 (2016).

40. J. F. Stephenson, M. Stevens, J. Troscianko, J. Jokela, The size, symmetry, and color saturation of a male guppy's ornaments forecast his resistance to parasites. The American Naturalist 196, 000-000 (2020).

41. J.-G. J. Godin, H. E. McDonough, Predator preference for brightly colored males in the guppy: a viability cost for a sexually selected trait. Behavioral Ecology 14, 194-200 (2003).

42. C. Van Oosterhout, P. Harris, J. Cable, Marked variation in parasite resistance between two wild populations of the Trinidadian guppy, Poecilia reticulata (Pisces: Poeciliidae). Biological Journal of the Linnean Society 79, 645-651 (2003).

43. D. M. Hawley, A. K. Gibson, A. K. Townsend, M. E. Craft, J. F. Stephenson, Bidirectional interactions between host social behaviour and parasites arise through ecological and evolutionary processes. Parasitology, 1-15 (2020).

44. H. Tao, L. Li, M. C. White, J. Steel, A. C. Lowen, Influenza A virus coinfection through transmission can support high levels of reassortment. Journal of Virology 89, 8453-8461 (2015).

45. J. F. Stephenson et al., Host heterogeneity affects both parasite transmission to and fitness on subsequent hosts. Philosophical Transactions of the Royal Society B: Biological Sciences 372, 20160093 (2017).

46. I. Eshel, Evolutionary and continuous stability. Journal of Theoretical Biology 103, 99111 (1983).

47. A. Hurford, D. Cownden, T. Day, Next-generation tools for evolutionary invasion analyses. Journal of the Royal Society Interface 7, 561-571 (2009). 
48. O. Leimar, Multidimensional convergence stability. Evolutionary Ecology Research 11, 191-208 (2009).

49. D. Reznick, M. Bryant, D. Holmes, The evolution of senescence and post-reproductive lifespan in guppies (Poecilia reticulata). PLoS Biol 4, e7 (2005).

50. J. F. Stephenson, Parasite-induced plasticity in host social behaviour depends on sex and susceptibility. Biology Letters 15, (2019).

51. S. Lopez, Acquired resistance affects male sexual display and female choice in guppies. Proceedings of the Royal Society of London. Series B: Biological Sciences 265, 717-723 (1998).

52. C. van Oosterhout et al., Selection by parasites in spate conditions in wild Trinidadian guppies (Poecilia reticulata). International journal for parasitology 37, 805-812 (2007).

53. F. Pérez-Jvostov, A. P. Hendry, G. F. Fussmann, M. E. Scott, Are host-parasite interactions influenced by adaptation to predators? A test with guppies and Gyrodactylus in experimental stream channels. Oecologia 170, 77-88 (2012).

54. A. E. Eiben, J. E. Smith, Introduction to evolutionary computing. (Springer, 2003), vol. 53.

55. R. Carnell, M. R. Carnell. (CRAN, 2016).

56. G. Pujol, B. Iooss, A. Janon. (CRAN, 2015).

57. S. W. Griffiths, A. E. Magurran, Sex and schooling behaviour in the Trinidadian guppy. Animal Behaviour 56, 689-693 (1998).

58. A. Magurran, B. Seghers, G. Carvalho, P. Shaw, Behavioural consequences of an artificial introduction of guppies (Poecilia reticulata) in N. Trinidad: evidence for the evolution of anti-predator behaviour in the wild. Proceedings of the Royal Society of London. Series B: Biological Sciences 248, 117-122 (1992).

59. C. Sievers et al., Reasons for the invasive success of a guppy (Poecilia reticulata) population in Trinidad. PloS one 7, e38404 (2012).

60. R. S. Mohammed et al., Parasite diversity and ecology in a model species, the guppy (Poecilia reticulata) in Trinidad. Royal Society Open Science 7, 191112 (2020).

61. A. M. Lyles, Genetic variation and susceptibility to parasites: Poeclia reticulata infected with Gyrodactylus turnbulli. (Princeton University, 1990).

62. B. A. Fraser, B. D. Neff, Parasite mediated homogenizing selection at the MHC in guppies. Genetica 138, 273 (2010).

63. D. Reznick, J. A. Endler, The impact of predation on life history evolution in Trinidadian guppies (Poecilia reticulata). Evolution, 160-177 (1982).

64. R. W. El $\square$ Sabaawi et al., Assessing the effects of guppy life history evolution on nutrient recycling: from experiments to the field. Freshwater Biology 60, 590-601 (2015).

65. N. Liley, P. Luyten, Geographic variation in the sexual behaviour of the guppy, Poecilia reticulata (Peters). Behaviour 95, 164-179 (1985).

66. D. N. Reznick et al., Eco-evolutionary feedbacks predict the time course of rapid lifehistory evolution. The American Naturalist 194, 671-692 (2019).

67. R. Xavier et al., Evidence for cryptic speciation in directly transmitted gyrodactylid parasites of Trinidadian guppies. PloS one 10, e0117096 (2015).

68. S. Kumar, G. Stecher, M. Li, C. Knyaz, K. Tamura, MEGA X: molecular evolutionary genetics analysis across computing platforms. Molecular biology and evolution 35, 1547 (2018).

69. M. Konczal et al., Gene duplications, divergence and recombination shape adaptive evolution of the fish ectoparasite Gyrodactylus bullatarudis. Molecular ecology 29, 14941507 (2020). 
70. M. Konczal et al., Expansion of frozen hybrids in the guppy ectoparasite, Gyrodactylus turnbulli. Molecular ecology 30, 1005-1016 (2021).

71. D. Wright, J. Krause, Repeated measures of shoaling tendency in zebrafish (Danio rerio) and other small teleost fishes. Nature Protocols 1, 1828-1831 (2006).

72. O. Friard, M. Gamba, BORIS: a free, versatile open $\square$ source event $\square$ logging software for video/audio coding and live observations. Methods in ecology and evolution 7, 13251330 (2016).

Acknowledgments: We thank Mahase Ramlal, David Reznick and Elizabeth Rudzki for assistance with fieldwork. Emmalina Calcaterra, Lindsay Colgan, Jukka Jokela, Maura Sackett, and Nadine Tardent provided technical assistance with parasite genotyping. Julie Johnson made artwork for Fig. 1. Jukka Jokela, Cock van Oosterhout, Martin Turcotte, and Kyle A. Young commented on an earlier version of this manuscript.

\section{Funding:}

National Science Foundation DEB: 2010826 (JCW)

National Science Foundation DEB: 2010741 (MJJ)

National Science Foundation DGE: 1747452 (FR)

University of Pittsburgh Central Research Development Fund (JFS)

\section{Author contributions:}

Conceptualization: JFS, JCW

Theoretical modeling: JCW, CEC, JFS

Data collection from literature: FR

Sensitivity analysis: JCW, FR

Field collections: MJJ, DRC, RP, RSM

Laboratory trait measurements: RP, DRC, MJJ, RDK, JFS

Parasite molecular work: MJJ, RDK, MK 
Parasite genetic analysis: MJJ, MK

Trait data analysis: JCW, DRC, JFS

Density and prevalence data analysis: JCW

Funding acquisition: JCW, MJJ, FR, JFS

Writing - original draft: JCW, JFS

Writing - review \& editing: JCW, MJJ, DRC, RDK, FR, RP, RSM, MK, CEC, JFS

Competing interests: The authors declare that they have no competing interests.

Data and materials availability: All data and code are available in the main text or the supplementary materials.

\section{Supplementary Materials}

Materials and Methods

Supplementary Text

Figs. S1 to S4

Tables S1 to S3

References (45-72)

Supplementary Code

Data files S1-S7 
Table 1. Meaning, value, and units for state variables, parameters, or outputs. Training values may be for a parameter, an output, or outputs related to a parameter or output. Training values were used in the model fitting and their sources are provided. Low/hi. pred. indicates predation regime. Values result from the model training. Note that the model was not trained with evolved virulence data but instead validated against it. See Materials and Methods for sources of training values.

\begin{tabular}{|c|c|c|c|}
\hline Symbol & Meaning & Value and/or units & Training value \\
\hline$S$ & Susceptible host density & hosts $\mathrm{m}^{-2}$ & \\
\hline 1 & Infected host density & hosts $\mathrm{m}^{-2}$ & \\
\hline$R$ & Recovered host density & hosts $\mathrm{m}^{-2}$ & \\
\hline$(S+I+R)_{\text {Low }}$ & Total host density, low pred. & 5.61 hosts $\mathrm{m}^{-2}$ & 8.19 \\
\hline$(S+l+R)_{\mathrm{Hi}}$ & Total host density, hi. pred. & 4.69 hosts $\mathrm{m}^{-2}$ & 4.68 \\
\hline $\begin{array}{l}(S+I+R)_{\mathrm{Hi}} / \\
(S+I+R)_{\mathrm{Low}}\end{array}$ & Host density ratio & 0.837 hosts $m^{-2}$ & 0.571 \\
\hline$p$ & Prevalence: $p=1 /\left(S_{+} l+R\right)$ & unitless & \\
\hline$p_{\text {Low }}$ & Prevalence, low pred. & 0.288 & 0.311 \\
\hline$p_{\mathrm{Hi}}$ & Prevalence, hi. pred. & 0.400 & 0.394 \\
\hline$p_{\mathrm{Hi}} / p_{\text {Low }}$ & Prevalence ratio & 1.39 & 1.27 \\
\hline$a$ & $\begin{array}{l}\text { Maximum host per-capita } \\
\text { fecundity }\end{array}$ & 0.106 day $^{-1}$ & $a=0.106$ \\
\hline$c$ & Host social contact rate & 1.7-5.5 $\mathrm{m}^{2}$ host $^{-1}$ day $^{-1}$ & \\
\hline$C_{\mathrm{H} i} / c_{\text {Low }}$ & Contact rate ratio & 2.23 & 1.93 \\
\hline$c_{1}$ & $\begin{array}{l}\text { Scaling parameter for contact } \\
\text { rate }\end{array}$ & $1 \mathrm{~m}^{2}$ host $^{-1}$ day $^{-1}$ & \\
\hline$d$ & Background host death rate & $1.30 \times 10^{-3}$ day $^{-1}$ & $d=1.41 \times 10^{-3}$ \\
\hline$k_{1}$ & $\begin{array}{l}\text { Trade-off parameter: Virulence } \\
\text { with transmissibility = } 1\end{array}$ & $1.38 \times 10^{6} \mathrm{day}^{-1}$ & See text \\
\hline
\end{tabular}




\begin{tabular}{|c|c|c|c|}
\hline$k_{2}$ & Trade-off parameter: Exponent & 3.61 & See text \\
\hline$P$ & Predation regime & $7.4-33.0 \times 10^{-3} \mathrm{day}^{-1}$ & \\
\hline$\left(d+\mathrm{Pc}_{1} / c+p v\right)_{\text {Low }}$ & Overall host mortality, low pred. & 0.011 day $^{-1}$ & 0.011 \\
\hline$\left(d+P c_{1} / c+p v\right)_{\mathrm{Hi}}$ & Overall host mortality, hi. pred. & 0.026 day $^{-1}$ & 0.026 \\
\hline $\begin{array}{l}\left(d+P c_{1} / c+p v\right)_{H i} / \\
\left(d+P c_{1} / c+p v\right)_{\text {Low }}\end{array}$ & Overall host mortality ratio & 2.46 & 2.47 \\
\hline$q$ & $\begin{array}{l}\text { Density dependence of host } \\
\text { fecundity }\end{array}$ & $0.017 \mathrm{~m}^{2}$ host $^{-1}$ day $^{-1}$ & \\
\hline$T$ & Transmissibility given contact & $6.2-8.81 \times 10^{-3}$ & Not trained \\
\hline$(c T)_{\text {Low }}$ & Transmission rate, low pred. & $0.015 \mathrm{~m}^{2}$ host $^{-1} \mathrm{day}^{-1}$ & 0.014 \\
\hline$c T I$ & Force of infection & day $^{-1}$ & Not trained \\
\hline$v$ & Mortality virulence & $0.015-0.053 \mathrm{day}^{-1}$ & Not trained \\
\hline$V_{\text {Low }}$ & Virulence, low pred. & 0.020 day $^{-1}$ & Validated: 0.021 \\
\hline$V_{\mathrm{Hi}}$ & Virulence, hi. pred. & 0.044 day $^{-1}$ & Validated: 0.041 \\
\hline$z$ & Rate of waning of host immunity & 0.033 day $^{-1}$ & $z=0.033$ \\
\hline$Y$ & Infected host recovery rate & 0.020 day $^{-1}$ & $y=0.033$ \\
\hline$\sigma$ & $\begin{array}{l}\text { Probability of successful } \\
\text { superinfection given } \\
\text { transmission }\end{array}$ & 1.21 & \\
\hline
\end{tabular}




\section{Materials and Methods}

\section{Molecular methods}

We used two methods of molecular species identification for individual parasites: we sequenced the mitochondrial COII gene for a subset of domestic lines established before March 2020, and we used a newly developed restriction enzyme assay for wild and domestic lines established after March 2020 (see Supplementary Text for further details). To prevent disrupting data collection, sample collection was restricted to already-dead hosts, stored in $70 \% \mathrm{EtOH}$. This conservative precaution meant we were unable to collect useable samples from some of our parasite lines.

We developed panels of single nucleotide polymorphisms (SNPs) for G. turnbulli and $G$. bullatarudis to examine the incidence of multi-genotype coinfections from wild collections, and to verify that the lines we established in the lab were genetically distinct. Genotypes were called using Fluidigm SNP genotyping analysis software. A subset of individuals was re-genotyped to identify and remove error-prone loci and estimate error rates (the proportion of mismatches to matches for re-genotyped loci). Loci which amplified consistently and with score calls greater than $90 \%$ were selected for use in the analysis. This resulted in a total of 140 variable loci for $G$. turnbulli (error rate 1.5\%) and 83 loci for G. bullatarudis (error rate $2 \%$ ) across all populations. However, due to significant local variation in informative loci, the total number of loci used for each source population varied (see Table S2). The resulting multilocus genotypes (MLGs) were assessed using GENALEX 6.502 to estimate the fraction of multi-genotype infections at six sites across Trinidad including our four focal populations (low- and high-predation in the Aripo and Guanapo rivers), and to assess the genotypes of the established lines.

As a conservative estimate, two individuals were considered to have different genotypes if they varied in at least $50 \%$ of the total variable loci from a given source population (see 
Supplementary Text for further method details). This applied both to determining that our lines were different genotypes and finding the frequency of multiple infections. In finding the frequency of multiple infections, we genotyped worms from fish with at least two worms and accounted for the proportion of fish with only one worm, estimated for each site from our 2020 survey and Stephenson et al. (28).

\section{Parasite trait data}

We established parasite lines by transferring a single worm to an uninfected host from our mixed, laboratory-bred stocks descended from wild populations. Each founding worm was obtained from a single guppy either from a commercial supplier (“domestic" lines) or wildcaught in Trinidad ("wild" lines from Caura, Aripo, Guanapo, and Lopinot rivers in Caroni drainage). Wild-caught adult guppies ( 50/population) were shipped from Trinidad to the University of Pittsburgh in March 2020 (see Animal use ethics statement). We established 43 parasite lines (18 domestic and 25 wild, 10 from low predation populations and 15 from high predation). Different lines were included in different analyses, as explained below. Lines were maintained under common garden conditions for 65 days (some domestic lines were maintained in the lab much longer) on groups of 3-6 guppies. We added uninfected fish to each line as required to replace those that either died or were found parasite-free during twice-weekly screening of all fish in each line under anaesthetic (tricaine methanesulfonate; $4 \mathrm{~g} / \mathrm{L}$ ) using a dissecting microscope. During these screens, we recorded the number of parasites infecting each fish. Each line was housed in a single 1.8 L tank on a recirculating system (Aquaneering Inc.; 12L:12D; $\left.24^{\circ} \mathrm{C}\right)$. Recirculated water passes through fine foam, sand, and ultraviolet filters before re-entering other tanks: individual parasites cannot transmit between lines, as supported by our genotyping. 
We estimated transmission rate by re-analysing data from Stephenson et al. (45) and conducting a similar new experiment. Donor fish received an infection of one parasite line or multiple infection and were individually housed in 1.8 L tanks [donor fish received line A, B, C, or A and B]; no lines in the new experiment were used by Stephenson et al. (45). We added a parasite-naïve recipient fish on day 8 of the donor's infection, and screened both fish for infection every $24 \mathrm{hrs}$.

We also assayed intensity and death rate. For intensity (a proxy for within-host growth rate), we counted the number of parasites on each host at each date (1 observation). We estimated the per-capita host death rate from the number of fish found dead divided by the number of infected fish in the tank at the previous observation time point and the days between observations ( 1 observation; $79 \%$ of observations were $3 \pm 1$ days after the previous one).

We calculated transmission rate from the number of days until successful infection of the recipient and intensity as the number of worms on the donor fish on the day of transmission, following Stephenson et al. (45) (total $n=101$ transmission events). Worm numbers changed slowly on the scale of the number of days until successful infection. In these assays, dS/dt = $c T_{i} I S$ ( $c$ is contact rate, $T_{\mathrm{i}}$ is the transmissibility of parasite line i, $I$ is infected host density, and $S$ is susceptible host density) so susceptible hosts follow an exponential decay pattern; thus, mean time until infection is $1 /\left(c T_{i} I\right)$. If days until transmission are $D$, then the transmission rate estimated by a given transmission event is $c T_{i}=1 /(D I)$. Based on the area of enclosures, infected host densities were $I=88.5$ hosts $\mathrm{m}^{-2}$ for Stephenson et al. $(45)$ and $I=72.0$ hosts $\mathrm{m}^{-2}$ for the follow-up experiment. We fit these estimates of transmission rate to $\log _{10}$ intensity with parasite line (line A, B, C, or coinfection of lines A and B) as a fixed effect using a generalized linear model with a Gamma error family and log link function. Parasite line was non-significant, so we refit the model without parasite line ( $\mathrm{P}$-value for intensity reported from this model; all $\mathrm{P}$-values 
represent two-sided, Type II Wald chi-square or F tests for this and other methods). Effect sizes are $\eta^{2}$ for ANOVA with only two levels of predictors, $\varphi$ for ANOVA with more than two levels of predictors, or partial correlation coefficients, $r$, for other analyses. For all analyses, we provide the code, output, and validation of the model fit in the Supplementary Code.

When determining average traits for a line, we only included lines maintained in the laboratory for more than 30 days $(n=22 ; 10$ domestic, 12 wild lines, including 1-4 lines from each of the four focal populations). We used an ANOVA to determine whether lines differed significantly in log intensity (1171 observations of intensity for the 22 parasite lines, at least 25 for each line). We modelled mean death rate for each line (344 observations and at least 13 for each line) as a function of mean intensity and line origin (wild $v s$. domestic) with a beta error family and logit link function. We estimated virulence $(v)$ by subtracting background death rate (see Table 1) from partial residuals of infected death rate (to get $v$ from $d+v$ ). We used partial residuals of infected death rate back-transformed onto the response scale to control for non-focal predictors (here, line origin) and find the relationship for wild parasites. We estimated mean transmission rate for a line by mapping the individual intensity measurements for each line onto the relationship observed between intensity and transmission in the experiments described above (Fig. 3A); we then calculated the mean transmission rate for each line. With estimates of transmission rate and virulence for each line, we examined the relationship between them using a generalized linear model of virulence with Gaussian error distribution and log link function as a function of natural $\log$ transmission rate. The statistical model was free to fit a positive (exponent $>1$ ), neutral (exponent $=1$ ), or negative curvature (exponent $<1$ ) to these data. We bootstrapped this fit by sampling lines to include in the analysis from the 22 parasite lines with replacement to determine how often the exponent was more than one. 
To examine the patterns in Fig. 5I and 5J, we used our estimates of parasite intensity and host death, (calculated as above) as response variables in GLMMs. These traits were assayed from parasites collected from low and high predation populations of our focal rivers (low/high predation; river distances: low Guanapo-high Guanapo $=11.1 \mathrm{~km}$, high Guanapo-high Aripo $=$ $23.9 \mathrm{~km}$, high Aripo-low Aripo = $6.6 \mathrm{~km} ; 3$ lines from high-predation Aripo and 5 from each of the other focal populations; 18 lines total). Each observation was of one line at one date but intensity on and death of multiple hosts for each line. This analysis included some lines maintained in the laboratory for less than 30 days but these lines necessarily had fewer observations and thus did not influence the analysis as much as lines with more observations. For intensity ( $N=425$ observations), we used predation regime, river, and number of fish in the common garden at time of measurement (since Gyrodactylus spp. can move relatively freely among fish) as fixed effects. Parasite line and lineday (days the line had been in the lab) nested within parasite line were included as random effects. We used $\log$ (intensity) as the response variable. For death rate $(N=216)$, we used predation regime and river as the fixed effects with parasite line and lineday nested within line as random effects, a Tweedie error family, and a log link function. The same analyses were used when determining the effect of predation regime on intensity $(N=208)$ and host death rate $(N=102)$ for known $G$. turnbulli lines. We compare the theoretical model to mean of back-transformed partial residuals of infected host death rate to control for non-focal predictors, such as lineday, to find the most representative estimate of death rate in each predation regime.

To determine trait differences by species, we considered all wild lines that were identified to species (including 7 from rivers considered non-focal due to lack of high predation $v s$. low predation comparisons). Three of these lines were G. bullatarudis and 11 were G. turnbulli. Because all three G. bullatarudis lines were from high predation populations, we only compared 
species differences within that regime. Species identity was used as a predictor instead of predation regime but statistical models were otherwise identical to those for the effect of predation regime for intensity $(N=345)$ and infected host death rate $(N=177)$.

\section{Theoretical modelling}

We wrote a relatively simple, ordinary differential equation model that retains the key biology of the predator-prey/host-parasite system. In the model, predators are not limited by prey/host density (e.g., because predators are generalists), allowing us to represent predation as a parameter $(P)$ for simplicity instead of a state variable. Susceptible hosts $(S)$ become infected $(I)$ and can recover to an immune state $(R)$ with waning immunity (see Table 1 for symbols, values, and units):

$$
\begin{gathered}
\frac{d S}{d t}=[a-q(S+I+R)](S+I+R)-\left(d+\frac{P c_{1}}{c}\right) S-c T I S+z R \\
\frac{d I}{d t}=c T I S-\left(d+\frac{P c_{1}}{c}\right) I-v I-\gamma I \quad(\mathrm{~S} 1 \mathrm{~B}) \\
\frac{d R}{d t}=\gamma I-\left(d+\frac{P c_{1}}{c}\right) R-z R \quad(\mathrm{~S} 1 \mathrm{C})
\end{gathered}
$$

Susceptible hosts are born from all hosts with density dependent, per-capita birth rate $a$ $q(S+I+R)$ (i.e., hosts grow logistically, eq. S1A). Hosts die at background rate $d$ and from predation at rate $P c_{1} / c$ that decreases with host shoaling rate $\left(c\right.$; that $P$ and $c_{1}$ together determine the strength of predation). Along with parasite transmissibility given contact $(T)$, the rate of density-dependent transmission from $I$ to $S$ depends on the host-host contact rate, dictated by shoaling rate. Recovered hosts also move back into the susceptible class as immunity wanes at rate $z$ (average duration of immunity is $1 / z$ ).

Infected hosts (eq. S1B) suffer background mortality and predation (predation is not selective in this model) while suffering additional mortality due to virulence, $v$. If predation was 
selective, especially to remove the sickest hosts, the consumptive effects of predation would be even more likely to select against virulence (3), strengthening our conclusion that shoaling rate drives virulence. Infected hosts recover with rate $\gamma$ (eq. S1C). Recovered hosts suffer background mortality and predation while losing immunity over time.

We modelled parasite and host evolution via Adaptive Dynamics (46). Biologically, parasite evolution is rendered more complex by the presence of multiple lines as well as two phenotypically similar species; host evolution is also complex because shoaling rate has important genetic and plastic components (27). For both host and parasite, our model is agnostic regarding the basis of adaptation but simply examines competition between phenotypes based on fitness of a rare phenotype (i.e., invasion analysis). Each phenotype corresponds to an asexual genotype in the model. Fitness when invading depends on the traits of the mutant (m) and resident genotypes (r) for parasites (eq. S2A):

$$
\begin{gathered}
\frac{1}{I_{\mathrm{m}}} \frac{d I_{\mathrm{m}}}{d t}=c T_{\mathrm{m}} S_{\mathrm{r}}^{*}-d-\frac{P c_{1}}{c}-v_{\mathrm{m}}-\gamma+\sigma c T_{\mathrm{m}} I_{\mathrm{r}}^{*}-\sigma c T_{\mathrm{r}} I_{\mathrm{r}}^{*} \\
v_{\mathrm{i}}=k_{1} T_{\mathrm{i}}^{k_{2}} \quad(\mathrm{~S} 2 \mathrm{~B})
\end{gathered}
$$

The resident sets host densities at $S_{\mathrm{r}}$ and $I_{\mathrm{r}}$ which depend on its traits, $T_{\mathrm{r}}$ and $v_{\mathrm{r}}$ (linked by the trade-off in eq. S2B). Mutant fitness depends on transmission to susceptible hosts, background death, predation, virulence, recovery, and gains and losses due to superinfection (eq. S2A). In superinfection, parasites of one genotype take over a host already infected by another genotype. We assume that each genotype has the same probability of successful superinfection given transmission, $\sigma$. This assumption is likely conservative as the probability of successful superinfection is often expected to increase with virulence (4), which could amplify the impact of multiple infections to select for higher virulence. From parasite fitness when invading, we find the continuously stable strategy, 'CSS', (46) for parasite virulence; a CSS represents a trait value 
that can be reached by gradual trait changes but that cannot be invaded by rare phenotypes with slightly different traits.

For host fitness, we used the Next Generation Matrix technique (47) that accounts for fitness within each class and the rates of movement between classes (see Supplementary Code). In the model, host genotypes differ in their contact rates, $c_{i}$. An invading host genotype (contact rate $\left.c_{\mathrm{m}}\right)$ contacts the resident genotype $\left(c_{\mathrm{r}}\right)$ at rate $\operatorname{sqrt}\left(c_{\mathrm{m}} c_{\mathrm{r}}\right)$ (for the purposes of transmission and predation, eq. S3), following Bonds et al. (35). An invading genotype suffers crowding (-q term, eq. S3A) from all hosts. Only intersections of a host CSS curve and parasite CSS curve (Fig. 4) can be a coevolutionary stable point (coCSS). We confirmed that a potential coCSS is indeed a coCSS with the strong convergence stability criterion (48).

$$
\begin{gathered}
\frac{d S_{\mathrm{m}}}{d t}=\left[a-q\left(H_{\mathrm{r}}\right)\right]\left(H_{\mathrm{m}}\right)-\left(d+\frac{P c_{1}}{\sqrt{c_{\mathrm{m}} c_{\mathrm{r}}}}\right) S_{\mathrm{m}}-\sqrt{c_{\mathrm{m}} c_{\mathrm{r}}} T I_{\mathrm{r}} S_{\mathrm{m}}+z R_{\mathrm{m}} \\
\frac{d I_{\mathrm{m}}}{d t}=\sqrt{c_{\mathrm{m}} c_{\mathrm{r}}} T I_{\mathrm{r}} S_{\mathrm{m}}-\left(d+\frac{P c_{1}}{\sqrt{c_{\mathrm{m}} c_{\mathrm{r}}}}\right) I_{\mathrm{m}}-v I_{\mathrm{m}}-\gamma I_{\mathrm{m}} \quad(\mathrm{S} 3 \mathrm{~B}) \\
\frac{d R_{\mathrm{m}}}{d t}=\gamma I_{\mathrm{m}}-\left(d+\frac{P c_{1}}{\sqrt{c_{\mathrm{m}} c_{\mathrm{r}}}}\right) R_{\mathrm{m}}-z R_{\mathrm{m}} \quad(\mathrm{S} 3 \mathrm{C})
\end{gathered}
$$

Note: $H=S+I+R$. In the second alternate model, the rate of predation on the resident is $P c_{1} / c_{\mathrm{r}}^{x}$ and predation on the mutant is $P c_{1} /\left(c_{\mathrm{r}} c_{\mathrm{m}}\right)^{x / 2}$ where $0<x . x>1$ indicates that protection from predation more than doubles when shoaling rate doubles. $x<1$ would mean that protection from predation less than doubles when shoaling rate doubles.

We have empirical estimates of some, but not all, of the parameters of the eco-coevolutionary model. As such, we used a simple evolutionary algorithm (described below) to estimate all model parameters, using empirical parameter estimates and field estimates of model outputs (e.g., prevalence) to fit the model to the data. Importantly, we did not use empirical data on evolved virulence in the fitting process, i.e., model virulence is free to evolve along the 
virulence-transmissibility trade-off however best fits the other data; instead, we used virulence data to validate the model.

Many empirical estimates came from the literature (see below). We estimated maximum birth rate (a) from lifetime fecundity of guppies with high food (49) in the laboratory. We estimated background mortality in the absence of parasites and predators $(d)$ as the inverse of life expectancy in the laboratory (49). We estimated recovery rate $(\gamma)$ as the inverse of the time required for fish to clear infection in the laboratory $(50)$. We estimated the rate at which immunity wanes $(z)$ from the observation that fish were roughly half resistant (averaging across totally resistant, partially resistant, and not resistant) 21 days after initial infection (51). Our trait measurements provided estimates for the trade-off parameters (Fig. 3C) given an estimate of $d$.

For parameters where we lacked estimates [importantly $P c_{1}$ (which acts as one parameter) in low- and high-predation populations and the superinfection parameter, $\sigma$ ], we also fit the model's eco-coevolutionary outputs at low and high predation to estimates of such outcomes in the field. We converted recapture rates from a mark-recapture experiment (52) into instantaneous mortality rates to determine overall mortality rate in the field $\left(d+P c_{1} / c+p v\right)$ for both a high predation population and a low predation population. We found a transmission rate reasonable for low predation populations $\left(c T_{\text {Low }}\right)$ by fitting transmission rate to peak prevalence and timing of peak prevalence found for low predation Aripo guppies and parasites in a stream mesocosm (53). We also used estimates of shoaling rate, prevalence, and host density (see Analysis of shoaling rate, prevalence, and host density below). We were able to fit some outputs to estimates from low predation populations, high predation populations, and the ratio of the two (representing the impact of changing predation regime). For others, we only have estimates of one of these three. This scheme weights the model training toward quantities that are wellcharacterized. See Table 1 for estimates used. 
We optimized the model's fit to both these empirical parameter estimates and field-estimated model outputs with a simple evolutionary algorithm (54). We began with parameters at their estimated values (for $a, d, k_{1}, k_{2}, \gamma$, and $z$ ). Initial values of other parameters were chosen arbitrarily $\left[q=1 \times 10^{-2},\left(P c_{1}\right)_{\text {Low }}=1 \times 10^{-2},\left(P c_{1}\right)_{\mathrm{Hi}}=3 \times 10^{-2}, \sigma=0.5\right]$. Each parameter was mutated by a factor of $10^{\wedge} x$ where $x$ is a single sample from the normal distribution $N(0,0.05)$ to create a parameter set; each parameter set formed one strategy in the evolutionary algorithm. One un-mutated strategy and 299 mutated strategies were evaluated in one "generation" of the algorithm by their summed relative error from all inputs and outputs with known estimates: summed relative error of strategy $\mathrm{x}=\left|a_{\mathrm{x}}-a_{\mathrm{data}}\right| / a_{\mathrm{data}}+\ldots+\left|c T_{\mathrm{Low}, \mathrm{x}}-c T_{\text {Low, data }}\right| c T_{\text {Low, data. }}$ There are four constrained parameters, four unconstrained parameters, and 8 independent scored outputs (e.g., $p_{\text {Low }}$ is independent from $p_{\text {Hi }}$ but not $\left.p_{\mathrm{Hi}} / p_{\text {Low }}\right)$. The strategy with the lowest summed relative error was passed to the next generation of the algorithm along with 299 mutated versions of itself. This process continues for 20 generations of the algorithm, as the model had asymptotically converged to a fit, yielding the parameter values used for the model (see Table 1).

We performed a sensitivity analysis to determine how key outcomes responded to parameter values. We performed this analysis for coCSS parasite prevalence, host density, parasite virulence, host contact rate, overall host mortality, transmission rates, and the ratios of these quantities between high and low predation populations. We also examine two other key 'proportion change metrics' representing how key factors change between the two, focal predation levels. First was the proportion of total host mortality change with predation regime that is caused by increased parasite-induced mortality: $\left(p_{\mathrm{Hi}} v_{\mathrm{Hi}} p_{\mathrm{Low}} v_{\mathrm{Low}}\right) /\left[\left(d+p_{\mathrm{Hi}} v_{\mathrm{Hi}}+P_{\mathrm{High}} c_{1} / c_{\mathrm{Hi}}\right)-\right.$ $\left.\left(d+p_{\text {Low }} v_{\text {Low }}+P_{\text {Low }} c_{1} / c_{\text {Low }}\right)\right]$. Second was the proportion of change in coevolutionary virulence across predation regime that is via predator's non-consumptive effects (evolution of higher shoaling rate). We defined this as the strength of pathway 3 (negative) plus the strength of 
pathway 4 (positive) divided by the summed strength of all pathways (see Fig. 1); we averaged pathway strength at the low predation point and the high predation point together to get one strength for each pathway (see eq. S5 for details). To generate random parameter sets, we used Latin Hypercube sampling with the 'lhs' R package (55) and then computed the partial rank correlation coefficients of the parameters with respect to each model outcome of interest (coevolutionary outcomes and key proportion change metrics) for $5 \times 10^{3}$ runs using the $\mathrm{R}$ package 'sensitivity' (56). We provide the code for the model and sensitivity analysis in the Supplementary Code.

4. Analysis of shoaling rate, prevalence, and host density

We extracted estimates of shoaling rate, prevalence, and host density from the literature and supplemented them with our own, new data on prevalence and shoaling rate. We used mixed models to test if guppy shoaling behaviour, Gyrodactylus spp. prevalence, and guppy density differed between predation regimes. In these models we used predation regime as a fixed effect along with river and year as random effects. We only included rivers with paired estimates from high and low predation populations because of variation across rivers. Each population average for a given site+year was one data point in these analyses. For \% time shoaling ( 22 estimates, 4 rivers; 26, 57-59) and prevalence (107 estimates, 11 rivers; 28, 31, 52, 53, 60-62), we used a generalized linear mixed model beta regression with a logit link. For host density (23 estimates, 4 rivers; 63, 64-66), we used a linear mixed model.

\section{Animal use ethics statement}

All collections and fish handling protocols were approved by the University of Pittsburgh's Institutional Animal Care and Use Committee: protocol 18072155 and 21069471. The permit to collect and export guppies was granted by the Director of Fisheries in the Ministry of Agriculture, Land and Fisheries Division, Aquaculture Unit of the Republic of Trinidad and 
Tobago on 2/26/2020 (copy available on request). The United States Import/Export License for

live and preserved fish was issued by the U.S. Fish and Wildlife Service, Office of Law

Enforcement Permit number A107080 on February 21, 2020 for the shipment of live guppies to the University of Pittsburgh. Samples were declared and cleared via USFWS form 3-177 on $03 / 11 / 2020$.

\section{Supplementary Text}

1. Supplementary equations

Host and parasite each evolve until selection favors neither a decrease nor an increase in the value of the evolving trait; at such "singular points", the effect of an invader's trait on the invader's fitness ("selection gradient", abbreviated $G$ here) is zero. The relative simplicity of $G_{T}$ ( $G$ for parasite transmissibility) provides insight into parasite evolution (eq. S4A derived from eq. S2):

$$
\begin{gathered}
G_{T}=\frac{\partial}{\partial T_{\mathrm{m}}} \frac{1}{I_{\mathrm{m}}} \frac{d I_{\mathrm{m}}}{d t}=\frac{d+\frac{P c_{1}}{c}+k_{1} T_{\mathrm{r}}^{k_{2}}+\gamma}{T_{\mathrm{r}}}-k_{2} k_{1} T_{\mathrm{r}}^{k_{2}-1}+\sigma c I_{\mathrm{r}}^{*} \\
G_{T}\left(T_{\text {coCss }}, c_{\text {coCss }}, P\right)=0 \quad(\mathrm{~S} 4 \mathrm{~B})
\end{gathered}
$$

Eq. S4B holds at all singular points; CSS and coCSS points are subsets of singular points. Since $G_{T}$ is zero, the derivatives sum to zero (eq. S5A), allowing disentangling the consumptive and non-consumptive effects of predation on parasite evolution.

$$
\begin{gathered}
0=\frac{\mathrm{d} G_{T}}{\mathrm{~d} P}=\frac{\partial G_{\mathrm{T}}}{\partial T_{\text {coCss }}} \frac{\mathrm{d} T_{\text {coCss }}}{\mathrm{d} P}+\frac{\partial G_{\mathrm{T}}}{\partial c_{\text {coCss }}} \frac{\mathrm{d} c_{\text {coCss }}}{\mathrm{d} P}+\frac{\partial G_{\mathrm{T}}}{\partial P} \\
\frac{\mathrm{d} T_{\text {coCss }}}{\mathrm{d} P}=\frac{1}{-\frac{\partial G_{\mathrm{T}}}{\partial T_{\text {coCsS }}}}\left(\frac{\partial G_{\mathrm{T}}}{\partial c_{\text {coCss }}} \frac{\mathrm{d} c_{\text {coCsS }}}{\mathrm{d} P}+\frac{\partial G_{\mathrm{T}}}{\partial P}\right)
\end{gathered}
$$




$$
\begin{gathered}
\frac{\partial G_{\mathrm{T}}}{\partial c_{\text {cocss }}} \frac{\mathrm{d} c_{\text {cocss }}}{\mathrm{d} P}+\frac{\partial G_{\mathrm{T}}}{\partial P}= \\
\frac{1}{T_{\mathrm{r}}}\left[\frac{\mathrm{d} c_{\operatorname{coCsS}}}{\mathrm{d} P} \frac{\partial}{\partial c}\left(\frac{P c_{1}}{c}\right)+\frac{\partial}{\partial P}\left(\frac{P c_{1}}{c}\right)\right]+\sigma\left[\frac{\mathrm{d} c_{\operatorname{cocss}}}{\mathrm{d} P} \frac{\partial}{\partial c}\left(c I_{\mathrm{r}}^{*}\right)+\frac{\partial}{\partial P}\left(c I_{\mathrm{r}}^{*}\right)\right]
\end{gathered}
$$

Note that $\partial G_{T} / \partial T_{\text {coCss }}<0$ by the evolutionary stability condition of Adaptive Dynamics, so $1 /(-$ $\left.\partial G_{T} / \partial T_{\text {coCSS }}\right)$ acts as a positive scaling factor in eq. S5B. Eq. S5C shows the consumptive and non-consumptive effects of predation in four terms on the right-hand-side. The first term finds the non-consumptive effect of predation on shoaling rate, thus on non-parasite induced mortality, and thus on parasite evolution (pathway 3 in Fig. 1). The second term finds the consumptive effect of predation on non-parasite induced mortality and thus on parasite evolution (pathway 1). The third term finds the non-consumptive effect of predation on shoaling rate, thus on multiple infections, and thus on parasite evolution (pathway 4). The fourth term finds the consumptive effect of predation on multiple infections and thus on parasite evolution (pathway 2). It is trivial to convert pathway strengths for $\mathrm{d} T_{\text {coCss }} / \mathrm{d} P$ to $\mathrm{d} v_{\text {coCsS }} / \mathrm{d} P$, given the trade-off. Thus, this model allows partitioning of the strengths by which predation consumptively and non-consumptively affects parasite evolution.

This same reasoning also shows how predation and host shoaling rate influence virulence evolution by influencing infectious period. From eq. S4A, we derive eqs. S6A and S6B.

$$
\begin{aligned}
& \frac{\partial}{\partial c} G_{T}=\frac{1}{T_{\mathrm{r}}} \frac{\partial}{\partial c}\left[d+\frac{P c_{1}}{c}+k_{1} T_{\mathrm{r}}^{k_{2}}+\gamma+\sigma c T_{\mathrm{r}} I_{\mathrm{r}}^{*}\right] \\
& \frac{\partial}{\partial P} G_{T}=\frac{1}{T_{\mathrm{r}}} \frac{\partial}{\partial P}\left[d+\frac{P c_{1}}{c}+k_{1} T_{\mathrm{r}}^{k_{2}}+\gamma+\sigma c T_{\mathrm{r}} I_{\mathrm{r}}^{*}\right]
\end{aligned}
$$

Infected host density of the resident genotype $\left(I_{\mathrm{r}}{ }^{*}\right)$ is not expanded because its expression is long. Infections for an invading genotype are lost at rate $d+P c_{1} / c+v+\gamma+\sigma c T_{\mathrm{r}} I_{\mathrm{r}}{ }^{*}$; thus, the effect of predation and shoaling rate on $G_{T}$ is proportional to their effect on the rate at which infections are 
lost. From eq. S5B (or a similar expression, e.g., if $c$ is simply a fixed parameter that is influencing parasite evolution), change in $T_{\text {coCss }}$ is proportional to change in $G_{T}$. Thus, the effect of predation or shoaling rate on the rate of infection loss captures their effect on transmissibility and virulence evolution. This derivation holds for all parameters not part of the parasite trait tradeoff (i.e., not $k_{1}$ or $k_{2}$ ).

\section{Identifying parasites to species}

For the first, sequencing method for species ID, we extracted parasite DNA from three individuals per line with the Qiagen DNeasy Blood and Tissue (Qiagen, Inc., Valencia, CA). We amplified a $262 \mathrm{bp}$ fragment of the mitochondrial COII gene following Xavier et al. (67) with the addition of a final extension step of $72 \mathrm{C}$ for $7 \mathrm{~min}$. Amplified DNA from 5 five domestic lines were sent to the DNA Analysis Facility on Science Hill at Yale University (New Haven CT, USA) for Sanger sequencing. Consensus sequences of reads in both the forward and reverse directions were assembled and edited manually in Sequencher 3.0 (Gene Codes Corporation, Ann Arbor, Michigan, U.S.). Sequences were aligned to reference sequences obtained from GenBank (G. turnbulli GenBank accession KP164811, G. bullatarudis GenBank accession KP168403) in MEGA-X 10.0.5 (68).

The second method was a PCR-restriction fragment length polymorphism (RFLP) assay we developed to differentiate between the three Gyrodactylus species that commonly infect guppies in Trinidad. This assay uses SspI-HF® (NEB) enzyme to cut the amplified portion of the mitochondrial COII gene differently depending on the species: $G$. turnbulli remains uncut, $G$. bullatarudis has 2 bands approximately 190 and 80bp, and G. poeciliae has 3 bands of approximately 140, 70 and 40bp. The restriction reaction followed manufacturer recommendations with $10 \mathrm{ul}$ of PCR product incubated for 12 hours at $37^{\circ} \mathrm{C}$. Bands were then 
visualized using a 4\% agarose gel. Control G. turnbulli and G. bullatarudis PCR products, identified by Sanger sequencing, were used during the development of the assay and for every digest to ensure quality enzyme activity.

\section{Parasite single nucleotide polymorphism (SNP) genotyping and analysis}

We distinguished parasite genotypes using SNPs. To identify potential SNP loci, we used the resequencing data in Konczal et al. $(69,70)$ from: six $G$. turnbulli from each of the Aripo and Lopinot Rivers, and two from the Caura River; and two G. bullatarudis samples from the Aripo River, seven from the Lopinot, three from the Caura, and eight from the Santa Cruz River. Raw reads for $G$. turnbulli were mapped to a $G$. turnbulli reference genome derived from a domestic collection (J. Stephenson, unpublished data) and G. bullatarudis to the reference genome (GenBank accession no: GCA_012064415.1) using bwa mem with the default parameters. Duplicates were marked using Picardtools. SNP calling was performed for the 100 longest scaffolds using samtools mpileup. SNPs within $5 \mathrm{bp}$ of an indel, within $250 \mathrm{bp}$ of another SNP, with quality scores below 100, or with mean mapping quality lower than 30 were excluded from subsequent analysis. Biallelic SNPs that were heterozygous in both Lopinot and Aripo (but with observed heterozygosity lower than 75\%) were selected for subsequent primer design.

Primer design for the potential SNP loci was conducted by Fluidigm following their best practice recommendations. Two custom 96.96 dynamic arrays for each species were screened for reliable amplification and consistent SNP calling for 96 individual parasites from both wild and domestic parasites. PCR was performed on a Fluidigm 96.96 Dynamic Array (SNP chip) with the following PCR cycling conditions: $50{ }^{\circ} \mathrm{C}$ for $2 \mathrm{~min}, 70{ }^{\circ} \mathrm{C}$ for $30 \mathrm{~min}, 25^{\circ} \mathrm{C}$ for $10 \mathrm{~min}$ and $95^{\circ} \mathrm{C}$ for $5 \mathrm{~min}$, followed by four touchdown cycles $\left(95^{\circ} \mathrm{C}\right.$ for $15 \mathrm{~s}$, from $64{ }^{\circ} \mathrm{C}$ to $61{ }^{\circ} \mathrm{C}$ for 45 
$\mathrm{s}, 72{ }^{\circ} \mathrm{C}$ for $\left.15 \mathrm{~s}\right) 34$ additional cycles $\left(95^{\circ} \mathrm{C}\right.$ for $15 \mathrm{~s}, 60{ }^{\circ} \mathrm{C}$ for $45 \mathrm{~s}, 72^{\circ} \mathrm{C}$ for $\left.15 \mathrm{~s}\right)$, and a final cycle at $20{ }^{\circ} \mathrm{C}$ for $10 \mathrm{~s}$.

\section{Data extraction from the literature}

We extracted site-level measurements of guppy shoaling behavior, Gyrodactylus spp. prevalence, and guppy density from the literature using GoogleScholar. All searches included the terms "Trinidadian guppy*" and "Poecilia reticulata". For shoaling estimates (Fig. 5F), we added "anti-predator response/behavior", "shoal*", "fusion-fission dynamics", "social*", "school*", "upper", "lower", "high predation", "low predation". We targeted our focal rivers by adding "Aripo" and "Guanapo". We recorded river, site, predation regime, and the metric the authors of the study used to estimate guppy social behavior, and any error estimate given. To obtain literature estimates of prevalence (Fig. 5G), we added the terms "Gyrodactylus", "infection" and "prevalence", and recorded the river, site, predation regime, and Gyrodactylus spp. prevalence among wild guppies, as well as year the estimates were taken, and any error estimates given. For host density (Fig. 5H), we added "density", and recorded or extracted the estimates of the number of guppies per $\mathrm{m}^{2}$. Papers that were obtained for other site-level measurements (shoaling and prevalence) were also examined to see if they incidentally also recorded density. Papers that gave estimates of these metrics under controlled conditions for lab lines of guppies instead of wild guppies were excluded. Where researchers reported multiple estimates, we took the mean value of those measured in one location at one timepoint as a single, independent observation.

\section{Supplementing data from the literature}

In March 2020, we collected guppies from each of our four focal populations using seine nets, transported them to the field station and held them in pools $(80 \mathrm{~cm}$ diameter $)$ for 24 to 
48hrs $($ mean $=33.6 \mathrm{hrs})$ before assaying their shoaling tendency. We used a standard assay to assess the shoaling tendency of individual fish (71) and recorded each trial using GoPro cameras (HERO4). We allowed fish to acclimatize for 10 minutes before removing a partition separating the focal fish from a shoal tank (containing three non-focal individuals from the same population), waited 200s after this removal, and then recorded the proximity of the focal fish to the shoal for a further 10 minutes using Boris (v7.9.8; 72). All assays and behavioral recordings were conducted by a single observer blind to infection status and predation regime. We removed some fish from the analysis because of camera error, or because the fish did not move for the trial duration. Our final sample sizes for each population were: low-predation Guanapo: 4 females, 5 males; high-predation: 3 females, 3 males; low-predation Aripo: 14 females, 13 males; high-predation: 12 females, 14 males. We recorded fish weight, length, sex, and presence of Gyrodactylus spp. infection after its behavior assay, and tested for their effects, along with fish predation regime and river of origin, on \% time shoaling using a generalized linear mixed model (Beta error, logit link function). Date of trial, lighting level, and behavior enclosure were included as random effects.

We collected prevalence estimates in March 2020 by catching between 50 and 30 fish per site with a $1 \mathrm{~m} \times 1 \mathrm{~m}$ seine net, and screening those from the Caroni drainage for parasites as above at a nearby field station. 

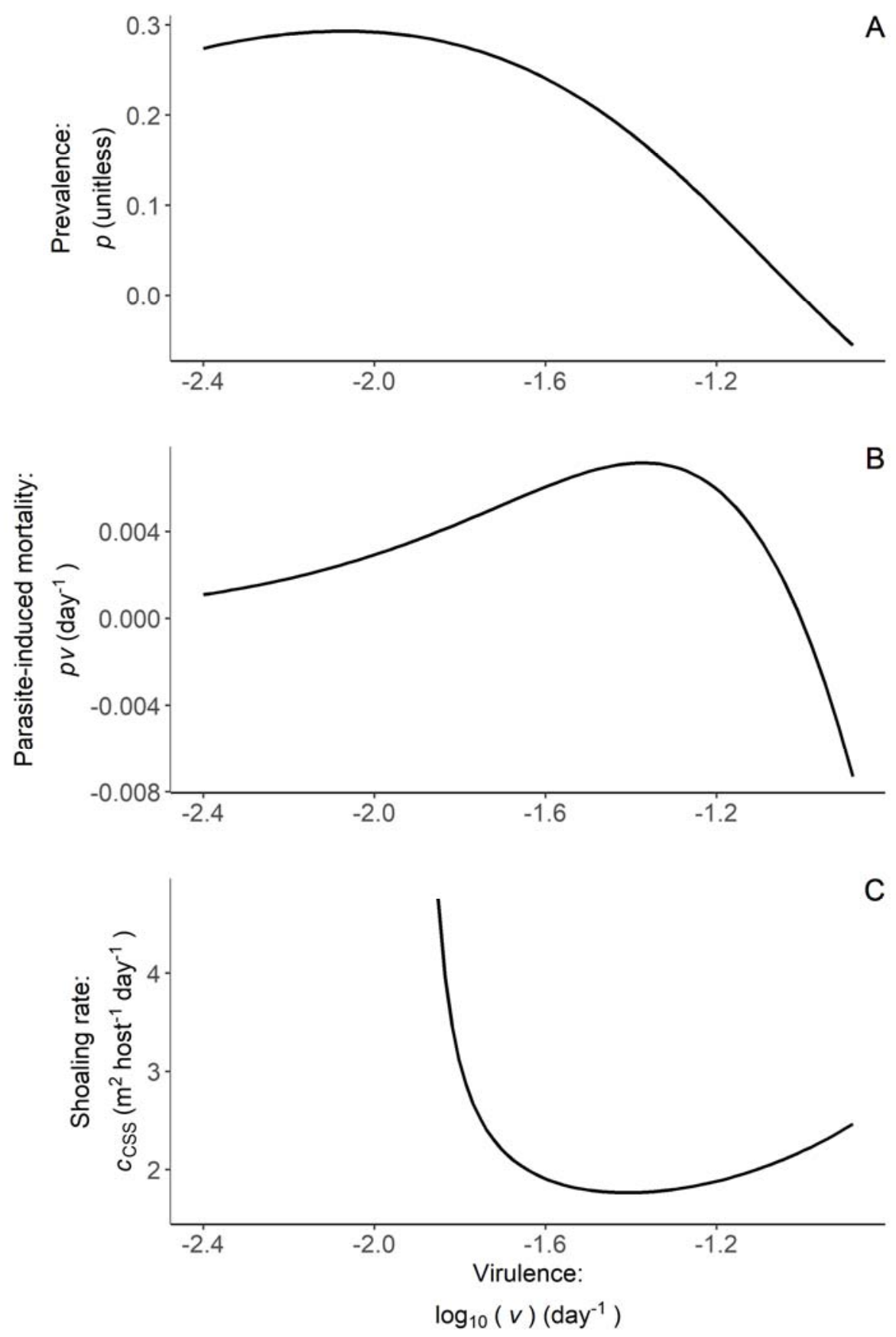

Figure S1. Virulence can select for increased contact rate. Hosts can evolve increasing contact rate in response to increased virulence, especially at very high virulence (beyond range used in main text). (A) Increasing virulence (and transmissibility along the trade-off) can decrease prevalence. (B) Overall parasite-induced mortality can decrease if prevalence declines sharply enough. This decrease occurs because, while parasites are very virulent, very few hosts 
are infected and suffering that virulence. $(\mathbf{C})$ At high virulence, increasing virulence can select

for higher host contact rates. $c=2$ used for (A) and (B); $P=0.074$ used for $(\mathrm{C})$. All other parameters at default (Table 1). 

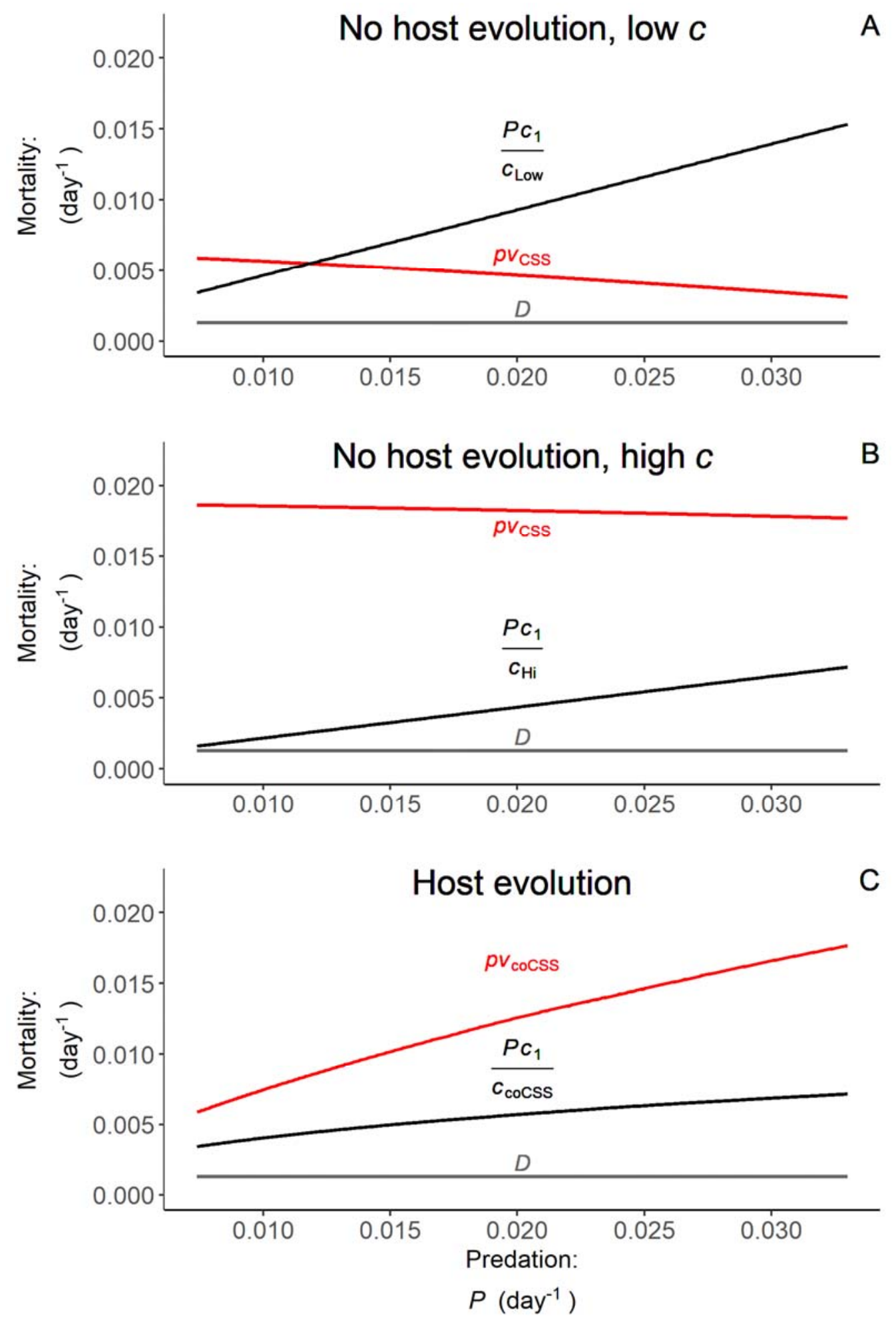

Figure S2. Host evolution in response to increasing predation causes parasite-induced mortality (red curves) to increase more than predator-induced mortality (black curves).

(A) Without host evolution (contact rate, c, set to the green point in Fig. 4 while parasites evolve 
to some CSS), parasite-induced mortality declines with predation while predator-induced mortality increases. Death from background sources ( $d$, grey line) does not change. (B) This trend is similar for a higher $c$ (set to high, blue point in Fig. 4). (C) When hosts evolve increasing $c$ with increasing predation (coCSS curve connecting green and blue points in Fig. 4), parasiteinduced mortality increases more than predator-induced mortality. This pattern is due to host evolution and is qualitatively unchanged if hosts evolve but parasites do not. 

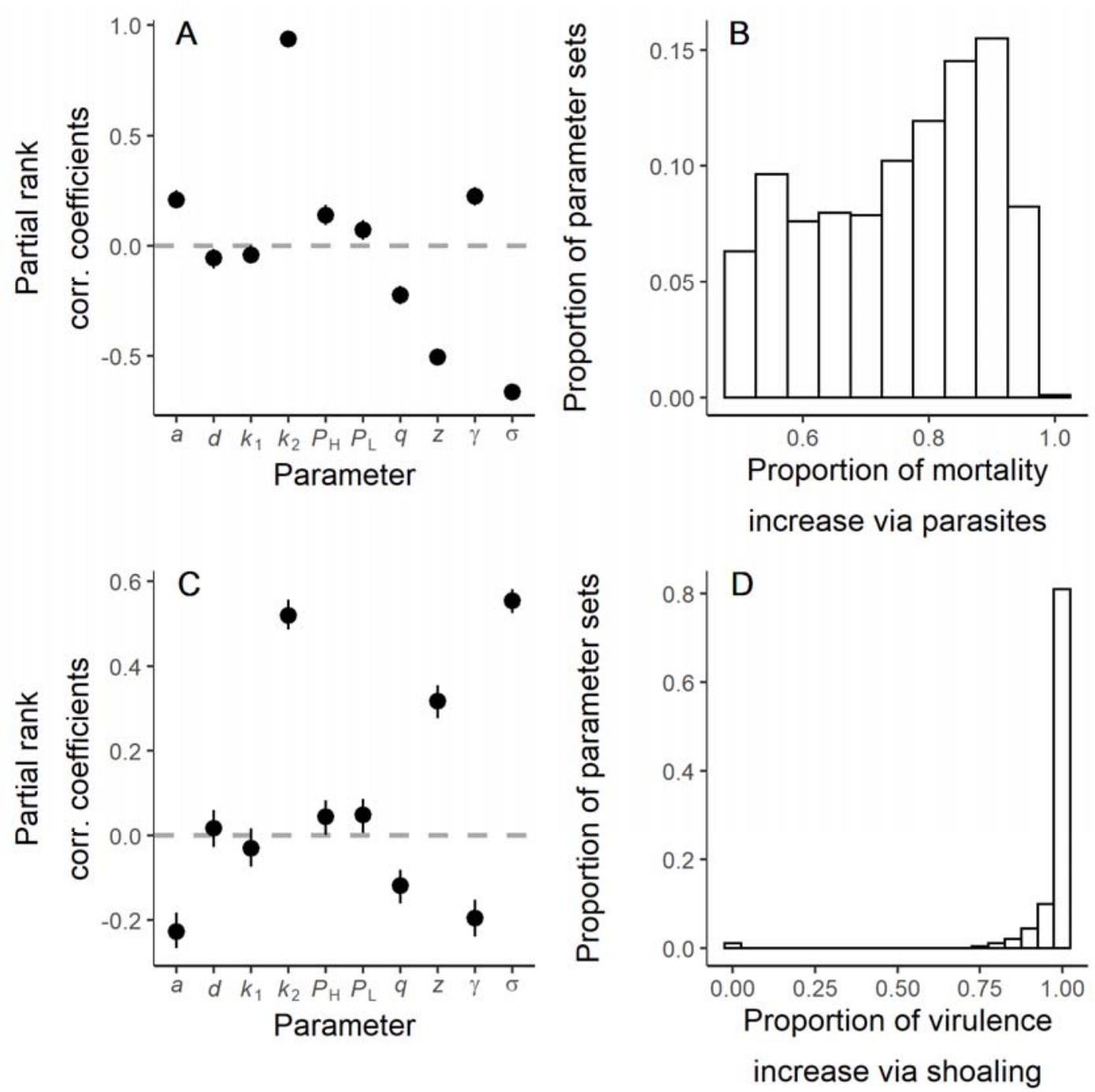

Figure S3. Sensitivity analysis shows the impact of parameters on key outputs and the range for those outputs. Key model outputs: the proportion of the mortality increase from increasing predation that is via increased parasite-induced mortality (A-B) and the proportion of the virulence increase from increasing predation that is via non-consumptive effects (C-D). Vertical lines are 95\% confidence intervals in (A \& C). (A) Notable among other parameters, the trade-off exponent, $k_{2}$, strongly increases the proportion of mortality increase due to parasites. Note here that $P_{\mathrm{H}}=P_{\mathrm{Hi}}$ and $P_{\mathrm{L}}=P_{\text {Low. }}$ (B) The proportion of mortality increase via parasites is 
typically well over half (0.5). (C) Both $k_{2}$ and the superinfection parameter, $\sigma$, strongly increase the proportion of virulence increase via non-consumptive effects. (D) The proportion of virulence increase via shoaling (i.e., non-consumptive effects of predators) is typically well over half. This proportion was less than 0.7 in only $1.10 \%$ of parameter sets because the net, nonconsumptive effects of predators on selection for virulence were negative in those parameter sets. 


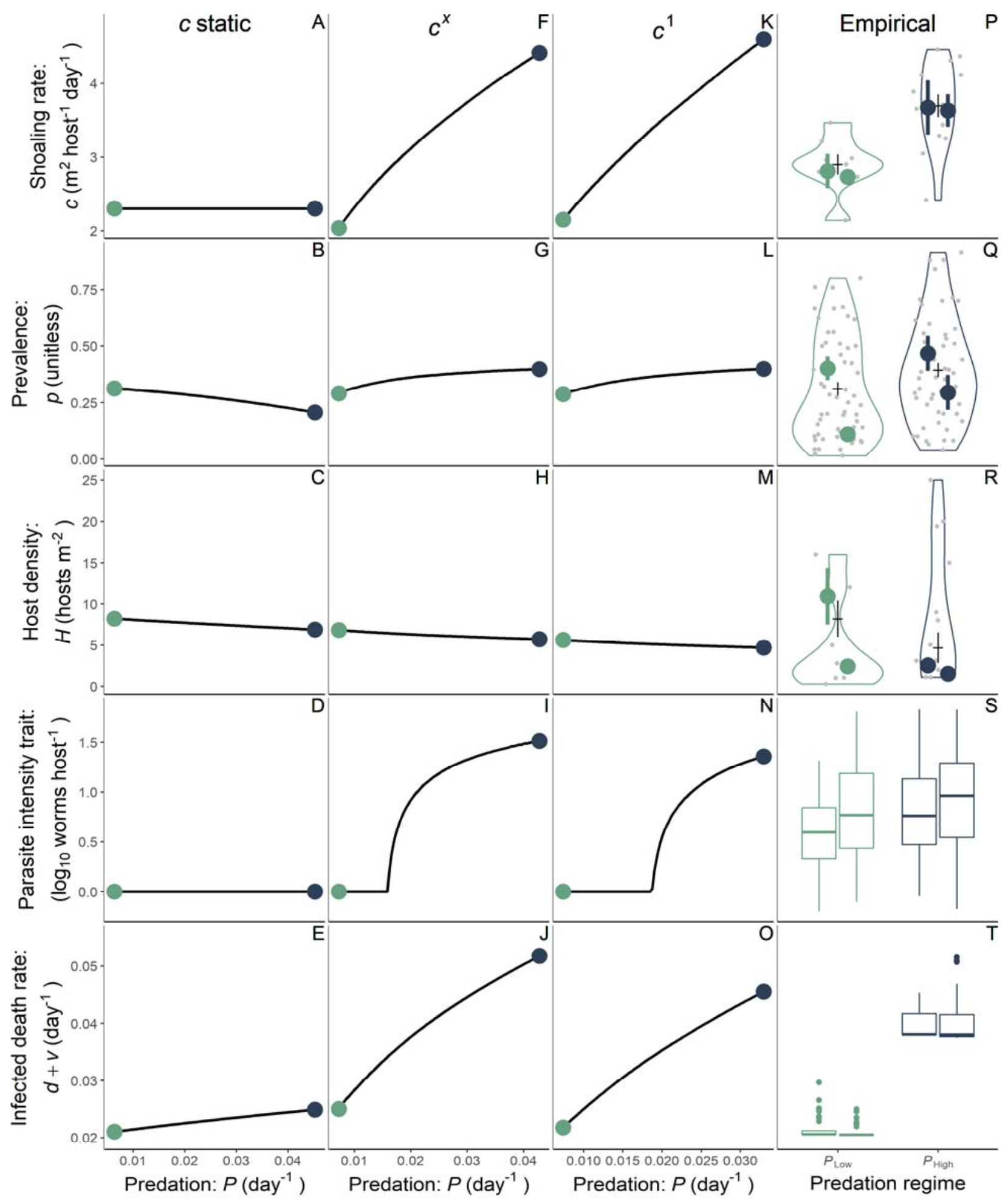

Figure S4. Alternate model fits to and predictions of data. Each of three models were allowed to fit as well as possible to the training data, e.g., by shifting the values of $P_{\text {Low }}$ and $P_{\mathrm{Hi}}$ (A-E) The model with $c$ fit as a static parameter, instead of output from host evolution, fits poorly to 
the data. Notably, it is a poor fit to prevalence, with prevalence decreasing with predation. (F-J) The model with an exponent governing the benefit of shoaling, $c^{x}$, fits the training data well but has little difference from the simpler model where the benefit of shoaling is simply $c, x=1.47 \mathrm{vs}$. $x=1$ in simpler model. (K-O) Accounting for model complexity, this model is best fits the training data such as (P-R) and thus is the focal model presented throughout. The appropriateness of this model is verified by the fact that it is also best at predicting the validating data $(\mathbf{S}-\mathbf{T})$. 
Table S1. Coinfection rates in the wild. For each site (river+predation regime), we genotyped multiple worms per a sample of fish hosting more than one worm. Coinfection within species $=$ (no. fish with $>1$ genotype of species $\mathrm{x} /$ no. fish with $>1$ worm of species $\mathrm{x}) \times \%$ of infections with $>1$ worm. Overall coinfection $=$ (no. fish with at least two different worms/no. fish with at least two worms) $\times \%$ of infections with $>1$ worm. Infections were diverse overall. Coinfection by identical multilocus genotypes was rare (31 out of the 73 worms possible). G.t. $=$ G. turnbulli and G.b. $=$ G. bullatarudis.

\begin{tabular}{|c|c|c|c|c|c|c|c|c|c|c|}
\hline \multirow{2}{*}{$\begin{array}{l}\text { Site } \\
\text { River }\end{array}$} & \multirow[b]{2}{*}{ Predation } & \multicolumn{2}{|c|}{ Sample size } & \multicolumn{3}{|c|}{$\begin{array}{l}\text { Species presence on } \\
\text { fish }\end{array}$} & \multirow{2}{*}{$\begin{array}{l}\text { Infections }>1 \\
\text { worm } \\
\%\end{array}$} & \multicolumn{2}{|c|}{$\begin{array}{l}\text { Coinfection within } \\
\text { species }\end{array}$} & \multirow{2}{*}{$\begin{array}{l}\text { Overall coinfection } \\
\text { rate } \\
\%\end{array}$} \\
\hline & & Fish & $\begin{array}{l}\text { Worms/fish } \\
\text { (mean } \pm S E)\end{array}$ & $\begin{array}{l}\text { \%G.t. } \\
\text { only }\end{array}$ & $\begin{array}{l}\text { \%G.b. } \\
\text { only }\end{array}$ & \%Both & & G.t. & G.b. & \\
\hline Aripo & High & 7 & $2.43 \pm 0.20$ & 14.3 & 85.7 & 0 & 70.8 & 0 & 35.4 & 30.3 \\
\hline Aripo & Low & 4 & $3.25 \pm 0.75$ & 100 & 0 & 0 & 58.2 & 14.6 & NA & 14.6 \\
\hline Guanapo & High & 7 & $2.29 \pm 0.18$ & 14.3 & 85.7 & 0 & 62.5 & 62.5 & 52.1 & 53.6 \\
\hline Guanapo & Low & 5 & 2 & 80 & 20 & 0 & 61.9 & 15.5 & 0 & 12.4 \\
\hline Santa & High & 7 & $4.86 \pm 1.30$ & 0 & 71.4 & 28.6 & 92.6 & 92.6 & 92.6 & 92.6 \\
\hline \multicolumn{11}{|l|}{ Cruz } \\
\hline Santa & Low & 3 & $3 \pm 1$ & 66.7 & 33.3 & 0 & 61.7 & 30.9 & 61.7 & 41.1 \\
\hline
\end{tabular}


Table S2. Threshold loci numbers. For each source population/species combination, a number of variable loci was found. To be considered a unique genotype, an individual had to differ from all other genotypes at half or more of the variable loci for that population. Some source populations only had one individual of a given species and thus have "NA" listed.

\begin{tabular}{|c|c|c|c|}
\hline Source population & Species & $\begin{array}{l}\text { Number of variable loci } \\
\text { within source }\end{array}$ & Number of loci threshold \\
\hline$P_{\mathrm{Hi}}$ Aripo & G. bullatarudis & 37 & 19 \\
\hline$P_{\mathrm{Hi}}$ Aripo & G. turnbulli & NA & NA \\
\hline$P_{\text {Low }}$ Aripo & G. bullatarudis & NA & NA \\
\hline$P_{\text {Low }}$ Aripo & G. turnbulli & 87 & 44 \\
\hline$P_{\mathrm{Hi}}$ Guanapo & G. bullatarudis & 22 & 11 \\
\hline$P_{\mathrm{Hi}}$ Guanapo & G. turnbulli & 46 & 23 \\
\hline$P_{\text {Low }}$ Guanapo & G. bullatarudis & 17 & 9 \\
\hline$P_{\text {Low }}$ Guanapo & G. turnbulli & 77 & 39 \\
\hline$P_{\mathrm{Hi}}$ Santa Cruz & G. bullatarudis & 41 & 21 \\
\hline$P_{\mathrm{Hi}}$ Santa Cruz & G. turnbulli & 23 & 11 \\
\hline$P_{\text {Low }}$ Santa Cruz & G. bullatarudis & 19 & 9 \\
\hline$P_{\text {Low }}$ Santa Cruz & G. turnbulli & 24 & 12 \\
\hline$P_{\mathrm{Hi}}$ Lopinot & G. turnbulli & 34 & 17 \\
\hline Commercial Source 1 & G. turnbulli & 31 & 16 \\
\hline Commercial Source 2 & G. turnbulli & NA & NA \\
\hline Commercial Source 3 & G. turnbulli & NA & NA \\
\hline
\end{tabular}


Table S3. Parameter effects based on $95 \%$ CIs. For each model output (first column), we report which parameters increased (second column) or decreased (third column) that model output in the sensitivity analysis. A positive or negative effect is only reported if the $95 \% \mathrm{CI}$ for that parameter's effect did not overlap 0. Trait outputs are the coCSS trait values.

\begin{tabular}{|c|c|c|}
\hline Model output & Parameters w. + effect & Parameters w. - effect \\
\hline$p_{\text {Low }}$ & $a, k_{2}, P_{\text {Low }}, z$ & $d, k_{1}, q, \gamma, \sigma$ \\
\hline$p_{\mathrm{Hi}}$ & $a, k_{2}, P_{\mathrm{Hi}}, z$ & $k_{1}, q, \gamma, \sigma$ \\
\hline$p_{\mathrm{H}} / p_{\text {Low }}$ & $d, k_{1}, P_{\mathrm{Hi}}, q, z, \gamma, \sigma$ & $a, k_{2}, P_{\text {Low }}$ \\
\hline$(S+l+R)_{\text {Low }}$ & $a, k_{1}, V$ & $k_{2}, P_{\text {Low }}, q$ \\
\hline$(S+I+R)_{\mathrm{Hi}}$ & $a, k_{1}, \gamma$ & $k_{2}, P_{\mathrm{Hi}}, q$ \\
\hline$(S+I+R)_{\mathrm{Hi}} /(S+I+R)_{\text {Low }}$ & $a, k_{1}, P_{\text {Low }}, q, z, \gamma, \sigma$ & $k_{2,}, P_{\mathrm{Hi}}$ \\
\hline$V_{\text {Low }}$ & $a, k_{2}, P_{\text {Low }}, \gamma, \sigma$ & $k_{1}, q, z$ \\
\hline$V_{\mathrm{Hi}}$ & $a, k_{2}, P_{H i}, \gamma, \sigma$ & $k_{1}, q, z$ \\
\hline$V_{\mathrm{HH}} / v_{\text {Low }}$ & $a, k_{2}, P_{\mathrm{Hi}}$ & $P_{\text {Low }}, \gamma, z, \sigma$ \\
\hline$c_{\text {Low }}$ & $k_{1}, P_{\text {Low }}, q, Y$ & $a, k_{2}, z, \sigma$ \\
\hline$C_{\mathrm{Hi}}$ & $k_{1}, P_{\mathrm{Hi}}, q, V$ & $a, k_{2}, z, \sigma$ \\
\hline$C_{\mathrm{HH}} / C_{\text {Low }}$ & $k_{2}, P_{\mathrm{Hi}}$ & $k_{1}, P_{\text {Low }}, q, z, \sigma$ \\
\hline$\left(d+P c_{1} / c+p v\right)_{\text {Low }}$ & $a, d, k_{2}, P_{\text {Low }}$ & $k_{1}, q, \gamma$ \\
\hline$\left(d+P c_{1} / c+p v\right)_{H i}$ & $a, k_{2}, P_{\mathrm{Hi}}$ & $k_{1}, q, \gamma, \sigma$ \\
\hline$\left(d+P c_{1} / c+p v\right)_{H_{i}} /\left(d+P c_{1} / c+p v\right)_{\text {Low }}$ & a, $q, P_{\mathrm{Hi}}, \gamma$ & $d, k_{2}, P_{\text {Low }}, \sigma$ \\
\hline Proportion of mortality increase via parasites & $a, k_{2}, P_{H i}, P_{\text {Low }}, Y$ & $d, q, z, \sigma$ \\
\hline$c T_{\text {Low }}$ & $k_{2}, P_{\text {Low }}, q, \gamma$ & $a, k_{1}, z, \sigma$ \\
\hline$c T_{\mathrm{Hi}}$ & $k_{2}, P_{\mathrm{Hi}}, q, \gamma$ & $a, k_{1}, z, \sigma$ \\
\hline$c T_{\text {Hil }} / c T_{\text {Low }}$ & $k_{2}, P_{\mathrm{Hi}}$ & $k_{1}, P_{\text {Low }}, q, z, \sigma$ \\
\hline Proportion of virulence increase via shoaling & $d, k_{1}, k_{2}, P_{\mathrm{Hi}}, P_{\text {Low }}, z, \sigma$ & $a, q, \gamma$ \\
\hline
\end{tabular}

